$11-1-2002$

\title{
JMASM4: Critical Values For Four Nonparametric And/Or Distribution-Free Tests Of Location For Two Independent Samples
}

Bruce R. Fay

Wayne County Regional Educational Service Agency, Michigan

Follow this and additional works at: http://digitalcommons.wayne.edu/jmasm

Part of the Applied Statistics Commons, Social and Behavioral Sciences Commons, and the Statistical Theory Commons

\section{Recommended Citation}

Fay, Bruce R. (2002) "JMASM4: Critical Values For Four Nonparametric And/Or Distribution-Free Tests Of Location For Two Independent Samples," Journal of Modern Applied Statistical Methods: Vol. 1 : Iss. 2 , Article 58.

DOI: $10.22237 /$ jmasm/1036110300

Available at: http://digitalcommons.wayne.edu/jmasm/vol1/iss2/58

This Algorithms and Code is brought to you for free and open access by the Open Access Journals at DigitalCommons@WayneState. It has been accepted for inclusion in Journal of Modern Applied Statistical Methods by an authorized editor of DigitalCommons@WayneState. 


\title{
JMASM Algorithms and Code \\ JMASM4: Critical Values For Four Nonparametric And/Or Distribution-Free Tests Of Location For Two Independent Samples
}

\author{
Bruce R. Fay \\ Assessment \& Evaluation \\ Wayne County Regional Educational Service Agency
}

Researchers engaged in computer-intensive studies may need exact critical values, especially for sample sizes and alpha levels not normally found in published tables, as well as the ability to control 'best-fit' criteria. They may also benefit from the ability to directly generate these values rather than having to create lookup tables. Fortran 90 programs generate 'best-conservative' (bc) and 'best-fit' (bf) critical values with associated probabilities for the Kolmogorov-Smirnov test of general differences (bc), Rosenbaum's test of location (bc), Tukey's quick test (bc and bf)) and the Wilcoxon rank-sum test (bc).

Key words: Kolmogorov-Smirnov test, Rosenbaum test, Tukey quick test; Wilcoxon rank-sum test.

\section{Introduction}

Researchers, especially those engaged in Monte Carlo studies, may have a need for exact critical values over a wider range of sample sizes and/or alpha levels than are generally available from published tables. They may also benefit from the ability to generate the values directly, as opposed to creating lookup tables, and to control best-fit criteria. Fortran 90 programs that generate critical values for four nonparametric/distribution-free tests of location for two independent samples are presented. Included are the Kolmogorov-Smirnov test of general differences, Rosenbaum's test of location, Tukey's quick test and the Wilcoxon rank-sum test. The programs for Tukey's test also generate 'best-fit' critical values and associated probabilities. The best-fit method could be adapted to the other programs.

Bruce R. Fay is an Assessment Consultant. He works with K-12 public schools in school accountability, accreditation, and assessment of student learning. Contact him at 30580 Springland St., Farmington Hills, MI 48334 or by e-mail at bfay@twmi.rr.com.

\section{Tukey Quick Test}

Tukey (1959) described a method for generating critical values for his Two-Sample Test to Duckworth's Specifications, now commonly known as Tukey's Quick Test. The test is both quick and compact, which makes it portable. The "rule of thumb" critical values, however, are not consistently 'best-conservative' or 'best-fit' to specific criteria.

\section{Test Description}

Tukey's (1959) test is quick in the sense that the method is easily remembered and the statistic, based on the combined length of extreme runs, easily calculated. The two samples are combined and ordered. For a two-sided test, if the overall maximum and minimum come from different groups, the statistic is the number of observations from the group with the global maximum that are greater than the greatest observation from the group with the global minimum plus the number of observations from the group with the global minimum that are less than the least observation from the group with the global maximum. If the global maximum and minimum are from the same group the statistic is generally taken to be zero. Tukey (1959) suggested dealing with ties (consequential, 
between-group) by counting each tied observation as $1 / 2$ rather than 1 . The one-sided (directional) test statistic is calculated just like the two-sided statistic with the additional requirement that the overall maximum observation is from the group that is expected to have the higher median under the alternative hypothesis (assuming a pure shift model). If not, the statistic is taken to be zero.

The test is compact in the sense that the critical values do not vary much with sample size, especially if the sample sizes are not too different. As such, they can also be easily committed to memory. For two-sided tests at nominal alpha levels of $.10, .05, .02$ and .01 (or one-sided tests at $.05, .025, .01$ and .005 ) the best-conservative critical values are 6, 7, 9 and 10 respectively with equal sample sizes from 9 to 24 per group. Tukey (1959) suggested that these critical values be used for all sample sizes as long as they were not too different. He noted, however, that under these conditions the test was not strictly conservative in the classical sense. He also gave relatively simple corrections to apply when the sample sizes were different, although not by too much. These corrections, however, still do not guarantee that the test will be strictly conservative, and add a level of complexity to the test that reduces both its quickness and compactness.

The best-fitting critical values for nominal alpha levels (1-sided) of .05, .025, .01, .005 (with a $+10 \%$ tolerance) are $6,7,8$ and 9 for equal samples sizes from 5 to 9 and $6,7,9,10$ for equal sample sizes from 11 to 30 . Using 6, 7, 9, 10 as the critical values for all equal sample sizes is conservative for samples sizes less than 11 at .02 and .01 alpha levels (2-sided) but may be liberal up to $+10 \%$ for other sample sizes and nominal alphas.

Quickness and compactness combine to make Tukey's (1959) test portable in the sense that everything needed to apply the test can be carried around in one's memory and the calculations can be performed mentally, or with pencil and paper. This simplicity is gained at the expense of some statistical power, but the practical power may be high. Tukey (1959) referenced a definition of practical power from Churchill Eisenhart (without formal citation) as "the product of the mathematical power by the probability that the procedure will be used" and noted that the practical power of a test might prove to be quite high, in spite of lower statistical power, if it became widely used.

Because of its portability and potentially high practical power, Tukey (1959) referred to this test as a "pocket test" and proposed that it filled a particular niche, i.e., "as a footrule", "on the floor", or "in the field" to "indicate the weight of the evidence roughly." $\mathrm{He}$ recommended that more sensitive tests be used "if a delicate and critical decision is to be made."

Methodology for Generating Critical Values and Associated Probabilities

Tukey (1959) described in detail a method for generating strictly conservative, exact critical values. That method is implemented in the program modules presented here, along with a variation that produces best-fitting critical values to a specified tolerance level above nominal alpha.

Tukey's (1959) method involves building a table, $A$, that contains "a certain summation of binomial coefficients." Differences of pairs of entries from $A$, based on the sample sizes $j$ and $k$ and a parameter $h$, are compared to ${ }_{n} \mathrm{C}_{j}$, the number of combinations of $n$ things taken $j$ at a time, where $n=j+k, j \geq 1, k \geq 1$, and $j \leq k$. The differences $A(k-h, j)-A(k, j-h)$ are formed starting with $h=1$ and counting up until the difference is less than (nominal alpha) $\mathrm{x}\left({ }_{n} \mathrm{C}_{j}\right)$. The first such value of $h$, if one exists, is the bestconservative critical value for that pair of sample sizes and nominal alpha level. Additional details of the method are given in the comments that accompany the programs. Based on the use of integer*8 and real*8 variables, critical values and associated probabilities are generated for all combinations of sample sizes from $(1,1)$ to $(30$, 30) in increments of 1 for each sample. Tukey (1959) also presented asymptotic methods that may be appropriate for larger sample sizes.

The module that generates the critical values and associated probabilities contains two versions of the method and a subroutine for calculating combinations. The first version of the method generates strictly conservative critical values for one-sided tests at $.05, .025, .01$ and .005 nominal alpha levels. The second version generates 'best-fit' critical values for one-sided tests at the same nominal alpha levels. The 'bestfit' version allows critical values greater than nominal alpha so long as they do not exceed 
nominal alpha by more than $10 \%$ and are closer to nominal alpha than the nearest value that is less than nominal alpha. The $+10 \%$ tolerance is based on a definition of robustness due to Bradley (1978).

\section{Rosenbaum's Test of Location}

Rosenbaum (1953, 1954) described tests for dispersion and location based on Wilks (1942) and gave tables of critical values. Rosenbaum (1965) revisited these tests, comparing them to other tests that had arisen in the intervening decade. Neave \& Worthington (1988) described the location form of the test as particularly well suited to situations in which spread is expected to increase with an increase in the median and gave a method for generating critical values. Their method is the basis for the programs presented here. Rosenbaum's (1954) test is quick and relatively compact, which makes it somewhat portable.

\section{Test Description}

The test is quick in the sense that the method is easily remembered and the statistic, based on the length of an extreme run, easily calculated. The two samples are combined and ordered. For a two-sided test, the statistic is taken as the number of observations from the group with the overall maximum that exceeds the maximum value of the other group. One way to deal with consequential (between-group) ties is to count each observation as $1 / 2$ rather than 1 . Another method is to average the values of the statistic arrived at by resolving the ties in all possible ways. The later technique, however, causes the test to lose some of its portability, at least for larger sample sizes. The one-sided (directional) test statistic is calculated just like the two-sided statistic with the additional requirement that the overall maximum observation is from the group that is expected to have the higher median under the alternative hypothesis (assuming a pure shift model). If not, the statistic is taken to be zero.

The test is compact in the sense that the critical values do not vary much with sample size, especially if the sample sizes are not too different. As such, they can also be easily committed to memory. For two-sided tests at nominal alpha levels of $.10, .05, .02$ and .01 (or one-sided tests at $.05, .025, .01$ and .005$)$ the best-conservative critical values are 5, 6, 7 and 8 respectively for equal sample sizes from 27 to 50 per group. Critical values of 5, 6, 7, and 8 can be used for equal sample sizes from 20 to 50 , and critical values of 4, 5, 6 and 7 for equal sample sizes from 5 to 19 , if one is willing to accept results that are not strictly conservative in all cases, and somewhat overly conservative in others. Under these conditions the test can be considered compact. Quickness and compactness combine to make the test portable as previously described.

Methodology for Generating Critical Values and Associated Probabilities

Neave \& Worthington (1988) described a method for generating strictly conservative, exact critical values. Their method is implemented in the program modules presented here to calculate the critical values for one-sided tests at $.05, .025, .01$ and .005 nominal alpha levels.

Neave \& Worthington (1988) calculated the probability of a run of $h$ values from a sample of size $m$ out of a combined sample of size $N=m$ $+n$, where $\mathrm{n}$ is the size of the other group, using the formula:

$$
\frac{m !(N-h) !}{N !(m-h) !}=\frac{m}{N} \times \frac{m-1}{N-1} \times \cdots \times \frac{m-h+1}{N-m+1} .
$$

The value of $h$ associated with the largest such probability that is less than or equal to nominal alpha is the critical value for a given $m$ and $n$. Thus all critical values are best-conservative with $\operatorname{pr}(\mathrm{CV}) \leq$ nominal alpha. Additional details of the method are given in the comments that accompany the programs. Based on the use of integer* 8 and real*8 variables, critical values and associated probabilities are generated for all combinations of sample sizes from $(1,1)$ to $(50,50)$ in increments of 1 for each sample.

Kolmogorov-Smirnov Test of General Differences Kim and Jennrich (1970, 1973) cited Smirnov (1939) as introducing the criterion $D_{m n}$ for the two-sample problem. As the name implies, the test is sensitive to general differences between two populations and is often used as a 2-sided test. Neave and Worthington (1988) pointed out, however, that the test functions quite well as a directional (1-sided) test, especially against a pure 
shift alternative. Kim and Jennrich (1970, 1973) provided a brief review of work on approximate and exact distributions of the statistic and resultant critical values under the null hypothesis leading up to their method and tables.

Test Description

The 2-sided test is conducted by constructing and then comparing the empirical cumulative distributions, $S_{m}(x)$ and $S_{n}(x)$, of two samples of size $m$ and $n$ ( $m \leq n$ without loss of generality) and then computing the criterion as $D_{m n}=\sup \left|S_{m}(x)-S_{n}(x)\right|$ over all $x$. The null hypothesis is that the two samples are drawn from identical (continuous) populations $F_{m}(x)$ and $F_{n}(x)$ (of any shape). The alternative hypothesis is that the samples were drawn from two populations that differ in some way. For a 1-sided test under a pure shift model, the criterion is taken to be $D_{m n}{ }^{+}$or $D_{m n}{ }^{-}$, where $D_{m n}{ }^{+}=\max \left[S_{m}(x)-S_{n}(x)\right] \geq 0$ and $D_{m n}{ }^{-}=\min \left[S_{m}(x)-S_{n}(x)\right] \leq 0$. The choice depends on which sample is presumed to come from the population with the higher median under the alternative hypothesis. If the alternative hypothesis is that the samples came from populations with cumulative distributions such that $F_{n}(x) \geq F_{m}(x)$ then $S_{n}(x)$ will lie to the right of $S_{m}(x)$. Thus, $S_{m}(x)$ will rise faster than $S_{n}(x)$ and lie above it for any given value of $x$. This makes $D_{m n}{ }^{+}$ the correct choice of criterion in this case.

Methodology for Generating Critical Values and Associated Probabilities

The Kim and Jennrich (1970, 1973) method of generating critical values for the Kolmogorov- Smirnov test is based on the work of Kim (1969) which, in turn, was an extension of the successive recursion relation of Massey (1951). Their method calculates:

$$
P\left(D_{m n} \leq \frac{c}{m n}\right)=U(m, n)
$$

where

$$
U(i, j)=\frac{i}{i+n} C(i, j)[U(i, j-1)+U(i-1, j)]
$$

and

$$
C(i, j)=\left\{\begin{array}{l}
1 \text { if }\left|\frac{i}{m}-\frac{j}{n}\right| \leq \frac{c}{m n} \\
0 \text { otherwise }
\end{array}\right.
$$

subject to initial condition

$$
U(i, j)=\left(\begin{array}{c}
i+n \\
i
\end{array}\right)^{-1} C(i, j), \text { when } i \bullet j=0
$$

Kim and Jennrich $(1970,1973)$ provided a FORTRAN IV function subroutine $\operatorname{ASKCDF}(M, N, D, U)$ that returned the probability of $D(=c / m n)$ for sample sizes $m$ and $n$ by calculating $U(m, n)$ as above. The $U$ referenced in their function subroutine argument list, however, was merely a working storage vector of at least length $N+1$. In the Fortran 90 implementation of $A S K C D F$ that follows, the working storage vector argument has been eliminated and replaced in the code with an allocatable array. A subroutine calculates $D=c / m n$ for $c=(1, m n, 1)$ for each combination of $n=(1,50,1)$ and $m=(1, n, 1)$ and calls $A S K C D F$ for each value of $D$ to obtain the probability and tests it against various nominal alpha levels.

\section{Wilcoxon Rank-sum Test}

Wilcoxon (1945) introduced the nonparametric/distribution-free test based on a sum of ranks that bears his name. Wilcoxon $(1946,1947)$ expanded on this work, followed by Mann and Whitney (1947), who described a test that turned out to be equivalent to the rank-sum test. The Wilcoxon-Mann-Whitney test is probably the best known of the nonparametric/distribution-free procedures. However, the early work of both Wilcoxon and Mann-Whitney provided only limited critical values. Additional work on both exact and approximate critical values and significance probabilities followed these seminal articles, e.g. Fix \& Hodges (1955).

Jacobson (1963) provided a nice synopsis of critical value tables and work-to-date with an extensive bibliography. Wilcoxon and Wilcox (1964, revised 1968) provided a workable method for generating critical values and probability levels. This work subsequently appeared in Wilcoxon, Katti and Wilcox (1970, revised 1973) 
and forms the basis for the programs presented here.

\section{Test Description}

The Wilcoxon rank-sum version of the test is conducted by combining the observations from two samples. The combined samples are then ranked while keeping track of the original group membership. The ranks from one of the groups are then summed to form the statistic. Which group to sum for a 1-sided test depends on the critical value tables that are available (lower-tail, upper tail, or both) and on which group is expected to have the least (or greatest) ranks under the alternative hypothesis. For example, if lower tail critical values are available, and the alternative hypothesis is that sample $B$ comes from a population that is greater than the population from which sample $A$ was obtained, then sample $A$ will tend to have the lower ranks, and the sum of those ranks would be taken as the statistic. For a two- sided test, one would form the sum of the ranks of both samples and test the resulting values against the critical value, taking the test to be significant if either comparison so indicated.

Methodology for Generating Critical Values and Associated Probabilities

Although critical values are readily available for the Wilcoxon rank-sum test and Mann-Whitney $U$ test, the probability levels are not as accessible. The method of Wilcoxon, Katti and Wilcox $(1970,1973)$ proceeds along the following lines given samples $M$ and $N$ from two continuous populations, $F_{m}(x)$ and $G_{n}(x)$ of size $m$ and $n$ respectively, $m \leq n$ without loss of generality. The minimum sum of ranks for sample $M$ is $m(m+1) / 2$. Thus the sum of ranks in general for sample $M$ is:

$$
\frac{m(m+1)}{2}+U \text { where } U \in I, U \geq 0
$$

The number of ways, $f(U)$, of obtaining a specific rank sum $U$, is the coefficient of $t^{U}$ in the expansion of the generating function, in powers of $t$, given by:

$$
g(t)=\prod_{i=1}^{n} \frac{\left(1-t^{m+i}\right)}{\left(1-t^{i}\right)}
$$

The total number of ways of obtaining any rank sum in this situation is:

$$
T=\left(\begin{array}{c}
m+n \\
n
\end{array}\right) .
$$

Given $F_{m}(x) \equiv G_{n}(x)$, the probability of obtaining $U$ is given by:

$$
\operatorname{pr}(U)=\frac{f(U)}{T}
$$

In turn, $f(\mathrm{U})$ can be found from:

$f(U)=\frac{1}{U} \sum_{i=0}^{U-1} f(i) z_{U-i-1}$

for $(U=1,2,3, \ldots)$ and with $f(0)=1$

In order to evaluate equation (10) it is necessary to find the values of $z$. Subroutine CV WRSJ4 init in module CVWRSJmod includes the code for generating the values of $z$.

Source Code and Computing Platforms

All source code provided here is Fortran 90 free format. For each of the four tests there is a module that contains the critical value generation subroutines and functions and a main program that can be used with that module to generate printed tables of critical values and probabilities. The programs were developed on a $500 \mathrm{MHz}$ AMD Athlon-based system using Compaq Visual Fortran 6.6 and tested on systems with Intel Pentium III and Pentium IV Xeon processors. The programs execute reasonably quickly on all of these systems. Even with integer*8 and real*8 variables these programs can run into arithmetic overflow problems, thus limiting the range of sample sizes for which critical values and probabilities can be generated. 


\section{References}

Bradley, J. V. (1978). Robustness? British Journal of Mathematical and Statistical Psychology, 31, 144-152.

Fix, E. and Hodges, J. L. Jr. (1955). Significance probabilities of the Wilcoxon test. Annals of Mathematical Statistics, 26, 301-312.

Jacobson, J. E. (1963). The Wilcoxon twosample statistic: Tables and bibliography. Journal of the American Statistical Association, 58, 10861103.

Kim, P. J. (1969). On the exact and approximate sampling distribution of the two sample Kolmogorov-Smirnov criterion $D_{m n}, m \leq$ n. Journal of the American Statistical Association, 64: 1625-1637.

Kim, P. J. \& Jennrich, R. I. (1970, 1973). Tables of the exact sampling distribution of the two-sample Kolmogorov-Smirnov criterion, $D_{m n}$, $m \leq n$. Selected Tables in Mathematical Statistics, Volume I (1970, $2^{\text {nd }}$ printing with revisions, 1973), 77-170. Harter, H. L. \& Owen, D. B., coeditors, Providence, RI: American Mathematical Society (edited by the Institute of Mathematical Statistics).

Mann, H. B. \& Whitney, D. R. (1947). On a test of whether one of two random variables is stochastically larger than the other. Annals of Mathematical Statistics, 18, 50-60.

Massey, F. J. Jr. (1951). The distribution of the maximum deviation between two sample cumulative step functions. Annals of Mathematical Statistics, 22, 125-128.

Neave, H. R. \& Worthington, P. L. B. (1988). Distribution-free tests. Unwin Hyman Ltd.

Rosenbaum, S. (1953). Tables for a nonparametric test of dispersion. Annals of Mathematical Statistics, 24, 663-668.

Rosenbaum, S. (1954). Tables for a nonparametric test of location. Annals of Mathematical Statistics, 25, 146-150.
Rosenbaum, S. (1965). On some twosample non-parametric tests. Journal of American Statistical Association, 60, 1118-1126.

Smirnov, N. V. (1939). Estimating the deviation between the empirical distribution functions of two independent samples. Bulletin de l'Universite' de Moscou, 2(2,3).

Smirnov, N. V. (1948). Table for estimating the goodness of fit of empirical distributions. Annals of Mathematical Statistics, 19, 279-281.

Tukey, J. W. (1959). A quick, compact, two-sample test to Duckworth's specifications. Technometrics, 1(1), 31-48.

Wilcoxon, F. (1945). Individual comparisons by ranking methods. Biometrics Bulletin, I(6), 80-83.

Wilcoxon, F. (1946). Individual comparisons of grouped data by ranking methods. Journal of Economic Entomology, 39(2), 269.

Wilcoxon, F. (1947). Probability levels for individual comparisons by ranking methods. Biometrics, 3, 119-122.

Wilcoxon, F. \& Wilcox, R. A. (1964). Some rapid approximate statistical procedures. Pearl River, NY: Lederle Laboratories Division, American Cyanamid Company. (Originally prepared and distributed in cooperation with the Department of Statistics, The Florida State University, Tallahassee, FL. and revised, 1968).

Wilcoxon, F., Katti, S. K., \& Wilcox, R. A. (1970, 1973). Critical values and probability levels for the Wilcoxon rank sum test and the Wilcoxon signed rank test. Selected Tables in Mathematical Statistics, Volume I (1970, $2^{\text {nd }}$ printing with revisions, 1973), 171-259. Harter, H. L. \& Owen, D. B., coeditors, Providence, RI: American Mathematical Society (edited by the Institute of Mathematical Statistics).

Wilks, S. S. (1942). Statistical prediction with special reference to the problem of tolerance limits. Annals of Mathematical Statistics, 13, 400409. 


\section{Programs}

\section{Tukey’s (1959) Two-sample Test to Duckworth’s Specifications (Tukey’s Quick Test)}

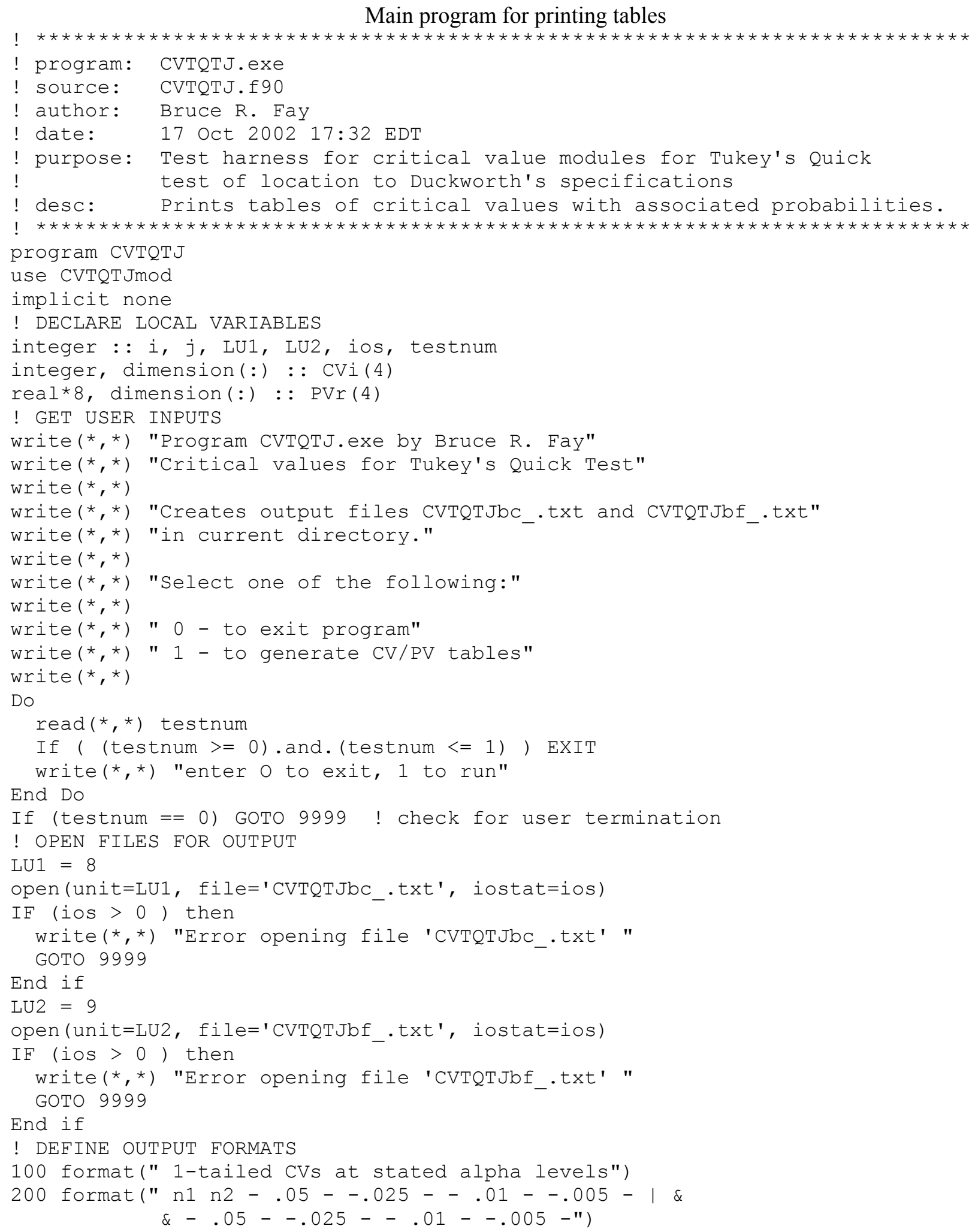




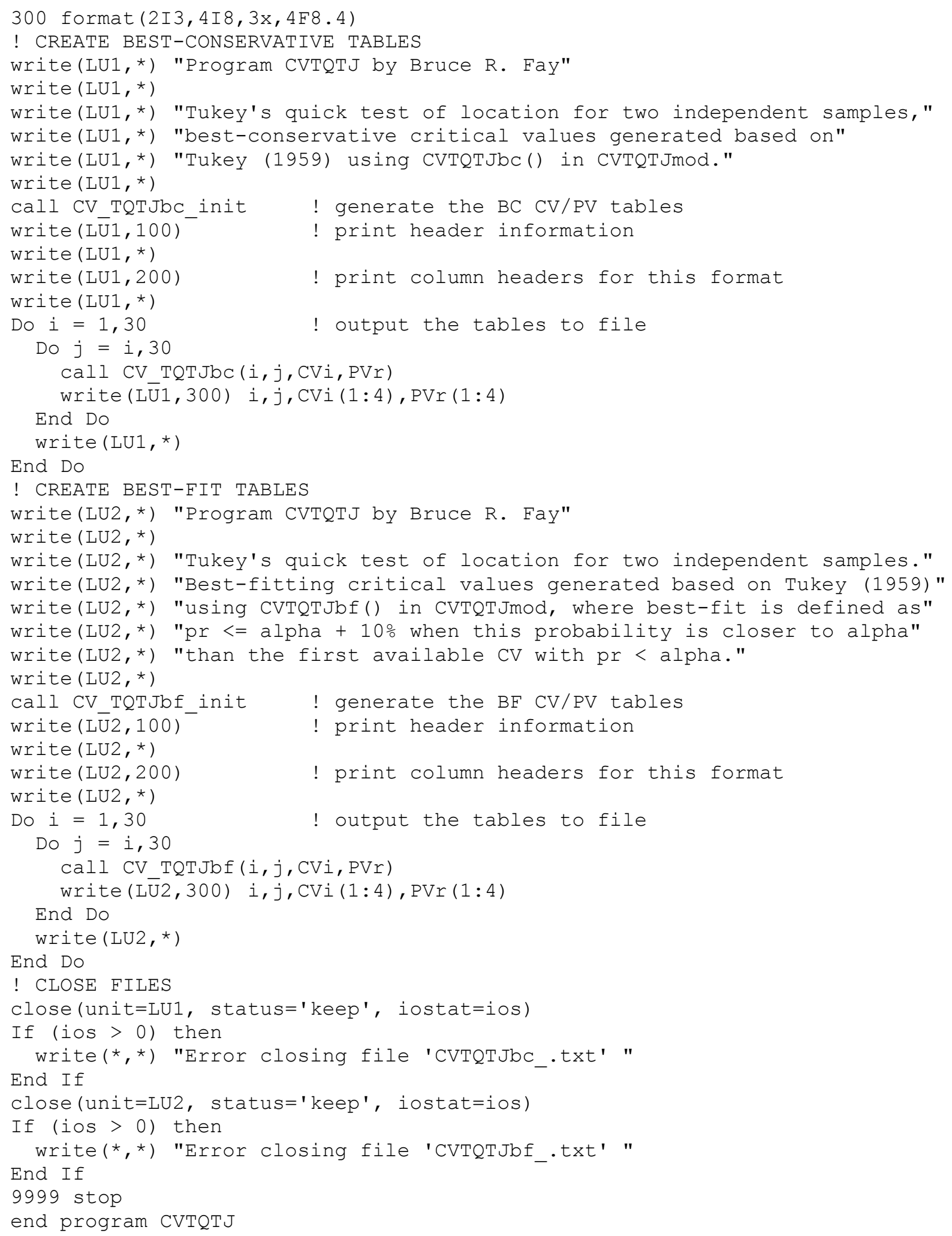




\section{Module for generating critical values and probabilities}

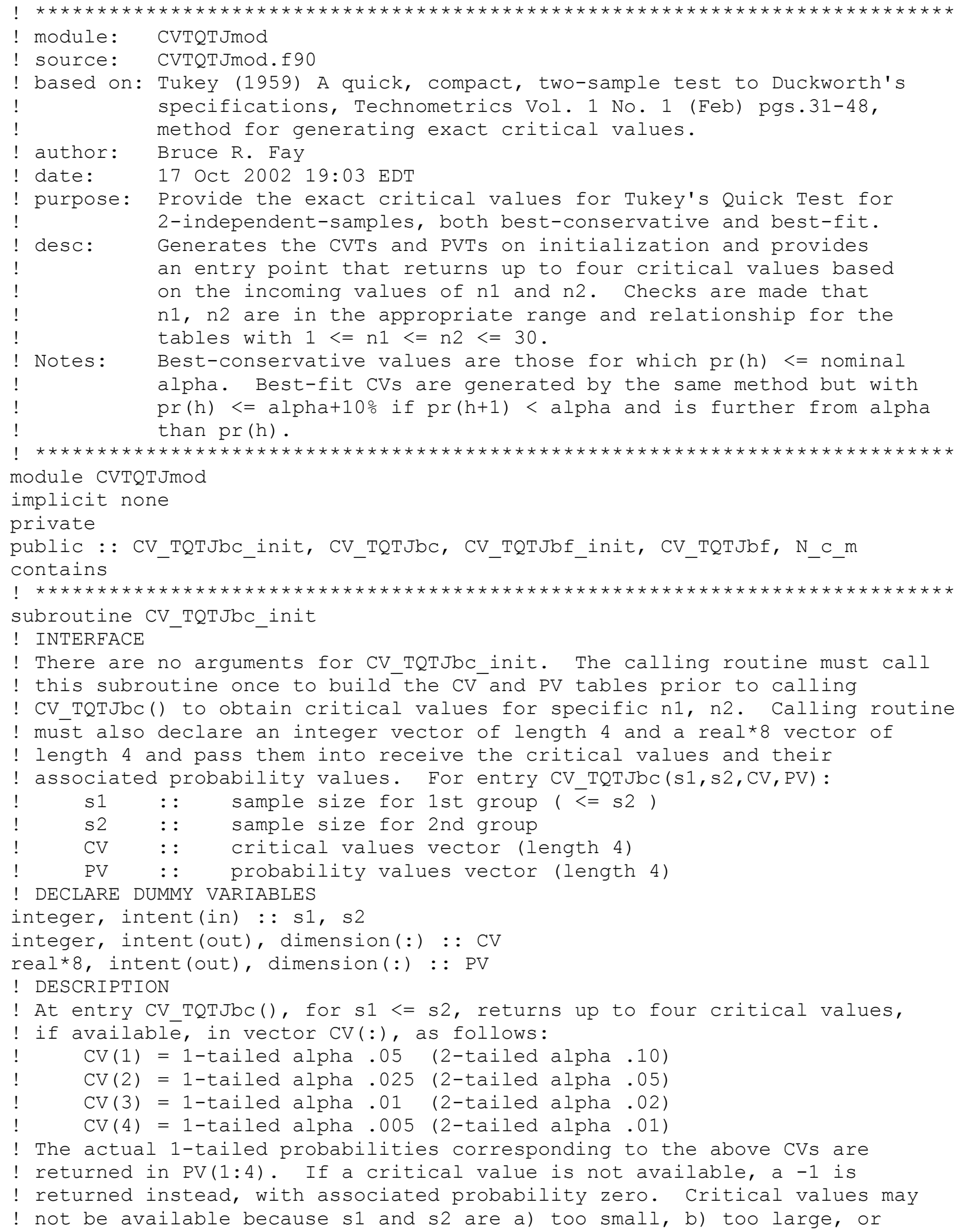


! c) too different. Unequal s1, s2 are supported for $1<=$ s $1<=$ s2 <= 30 . ! DECLARE LOCAL VARIABLES

integer:: $h, \mathrm{n} 1, \mathrm{n} 2, \mathrm{v} 1, \mathrm{v} 2, \mathrm{w} 1, \mathrm{w} 2$

integer $(\mathrm{kind}=8):: \mathrm{wv} 1, \mathrm{wV} 2$

integer (kind=8), dimension $(30,30)$, save :: CVTbc05, CVTbc025

integer (kind=8), dimension $(30,30)$, save : : CVTbc01, CVTbc005

integer (kind=8), dimension $(0: 30,1: 30)$, save : : Atbl

integer $(\mathrm{kind}=8):: \mathrm{comb}, \mathrm{A} 1, \mathrm{~A} 2$, Adiff

integer $(\mathrm{kind}=8)$, parameter : : zero=0, one=1, two=2

real (kind=8), dimension(30,30), save : : PVTbc05, PVTbc025, PVTbc01, PVTbc005

real (kind=8), parameter : : $m 05=0.050, m 025=0.025, m 01=0.01, m 005=0.005$

real (kind=8):: c05, c025, c01, c005, rcomb, rdiff

logical : : fnd05, fnd025, fnd01, fnd005

! Build the A table

!

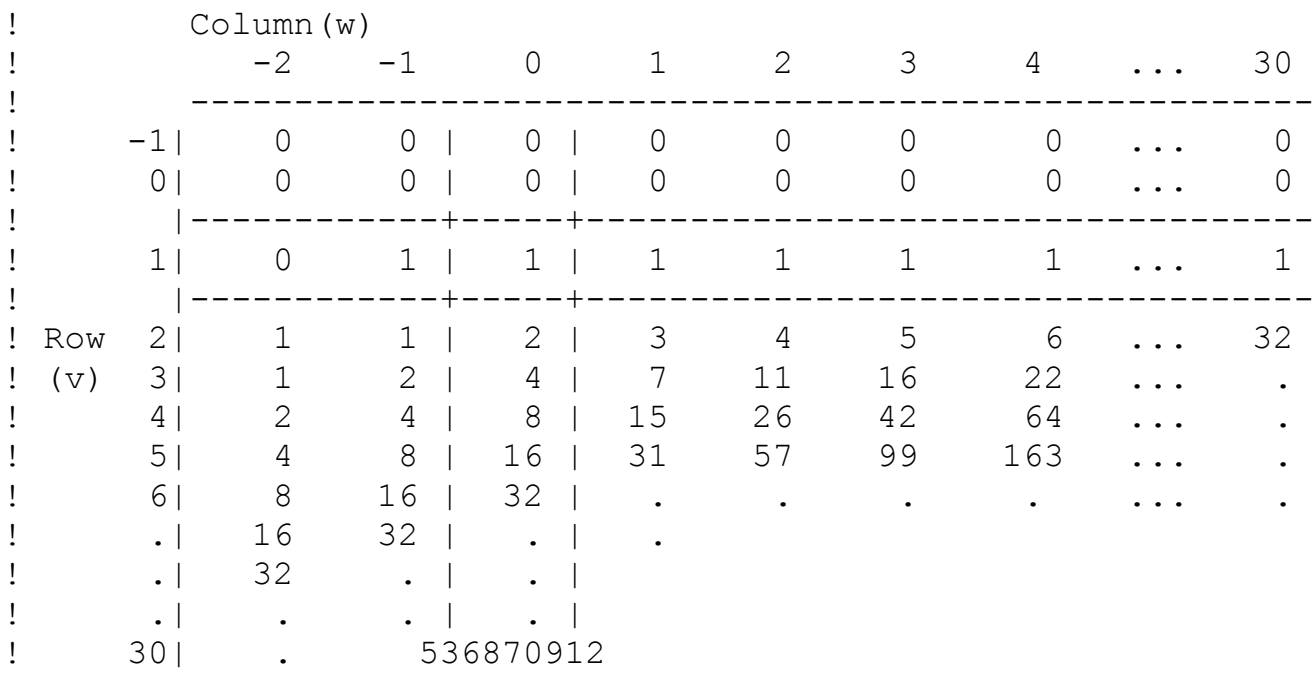

! Note: The A table is only built for columns 0 to 30 and rows 1 to 30 . All

! entries for rows less than one are zero and all entries for columns

! less than zero (with rows of 1 or more) can be determined by direct

! formula (see code).

Atbl $(0: 30,1)=$ one ! first row, all columns, entries $=1$

Do $\mathrm{V} 1=2,30$ ! first (zero) column, row entries are $2^{\wedge}($ row -1$)$

$\operatorname{Atbl}(0, \mathrm{v} 1)=\operatorname{two} *(\mathrm{v} 1-1)$

End Do

Do $\mathrm{v1}=2,30 \quad$ ! previous column same row + same column previous row

Do $\mathrm{w} 1=1,30$

End Do

Atbl $(w 1, v 1)=A t b l(w 1-1, v 1)+A t b l(w 1, v 1-1)$

End Do

CVTbc05 = -1 ! initialize the CV tables to -1 (indicates no valid entry)

CVTbc025 $=-1$

CVTbc01 $0-1$

CVTbc005 $=-1$

PVTbc05 = 0.0 ! initialize the PV tables to 0.0 (indicates no valid entry)

PVTbc025 = 0.0

PVTbc01 $=0.0$

PVTbc005 = 0.0

! Determine the critical values and associated actual probabilities

Do $\mathrm{n} 1=1,30 \quad ! \mathrm{n} 1$ for $\mathrm{CV} / \mathrm{PV}$ tables

Do $\mathrm{n} 2=\mathrm{n} 1,30$ ! $\mathrm{n} 2$ for $\mathrm{CV} / \mathrm{PV}$ tables 


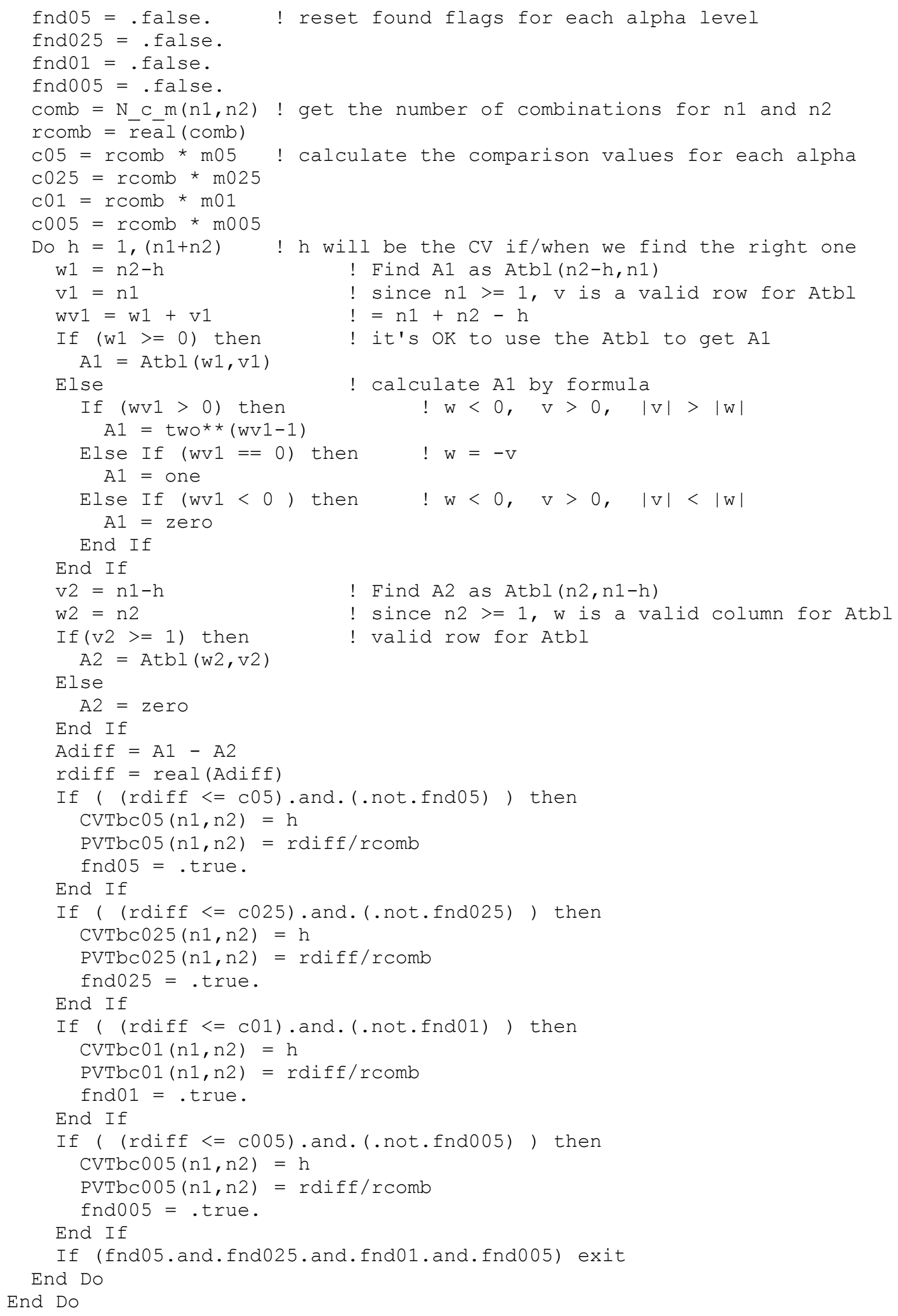




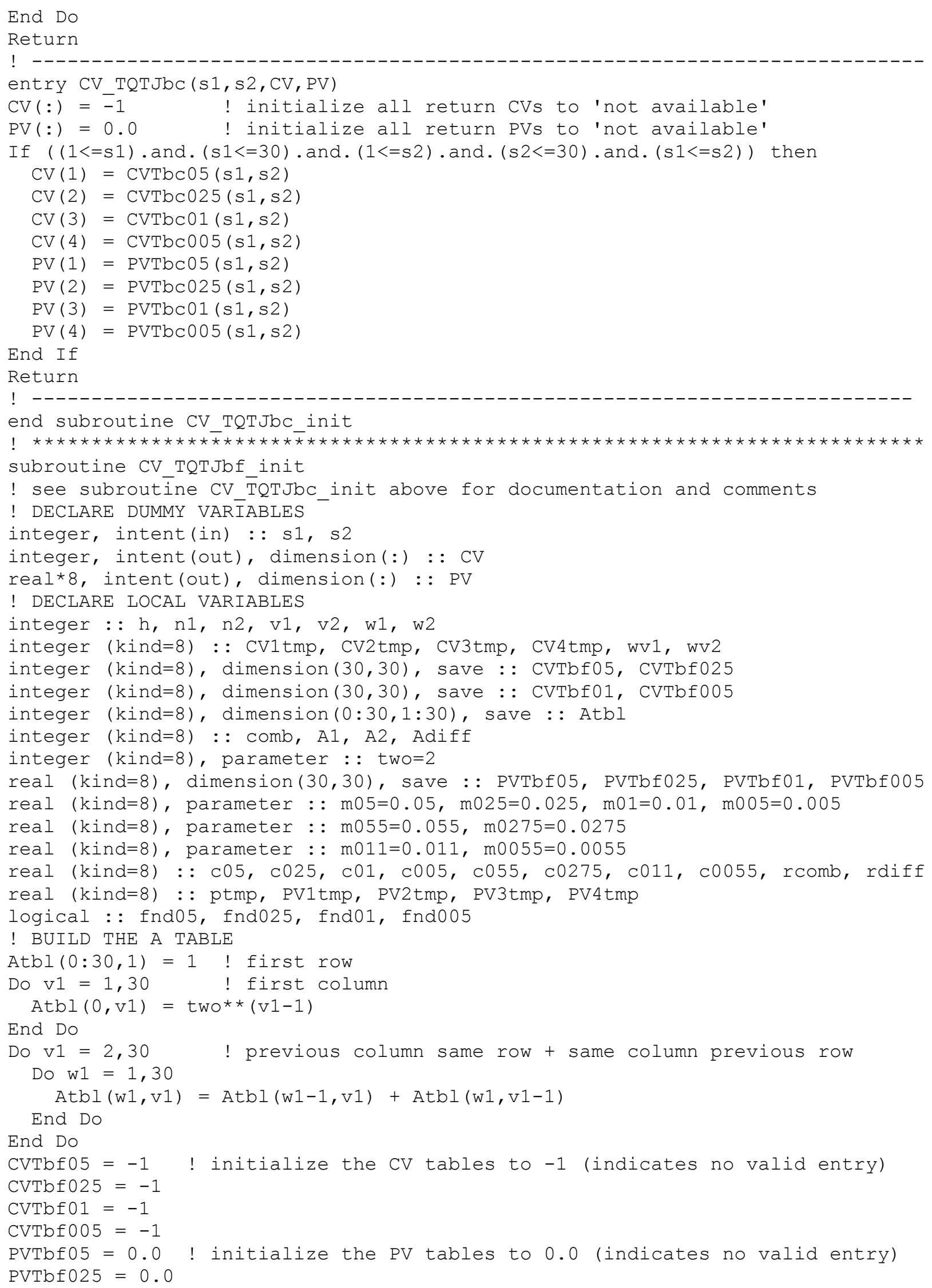




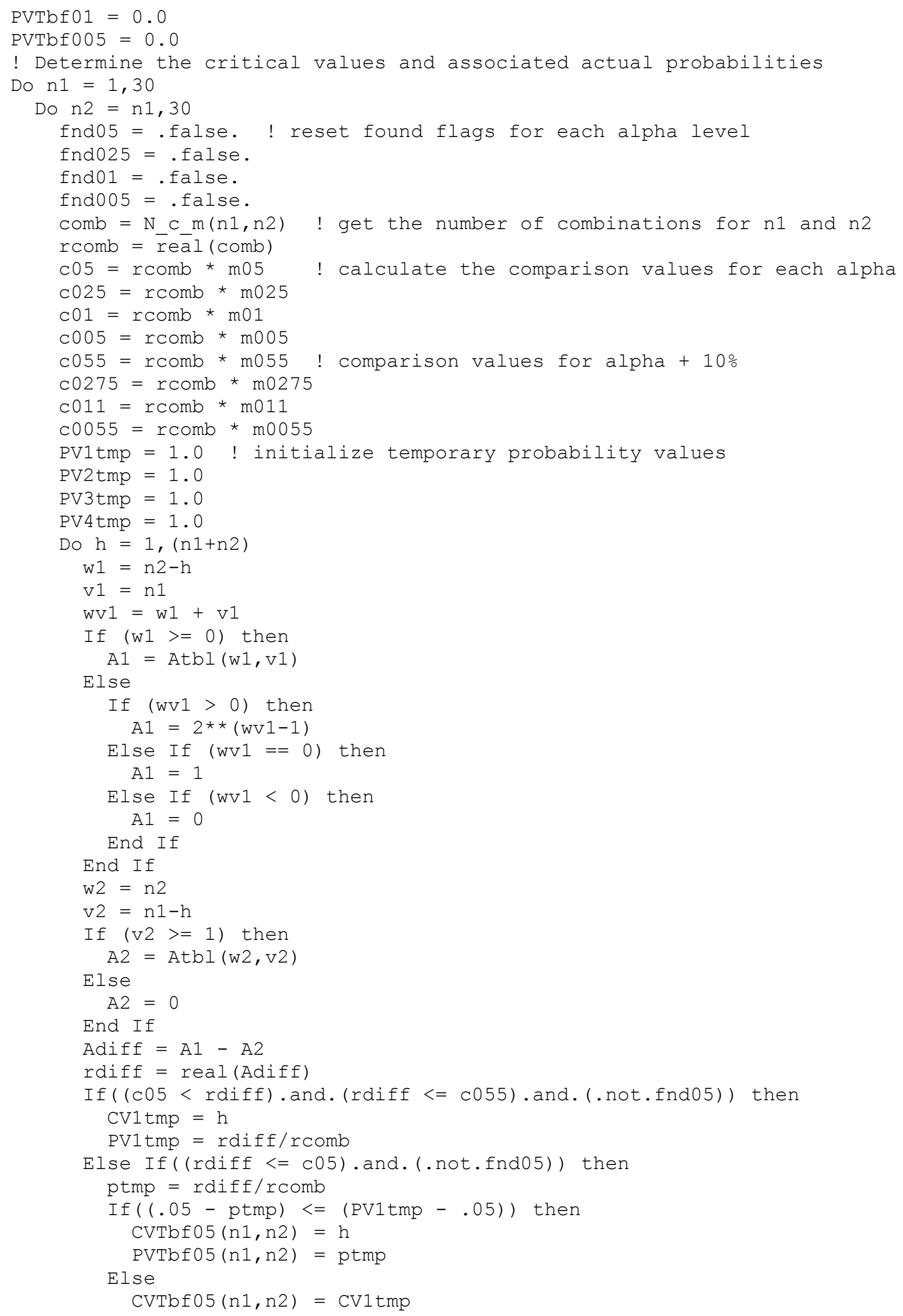




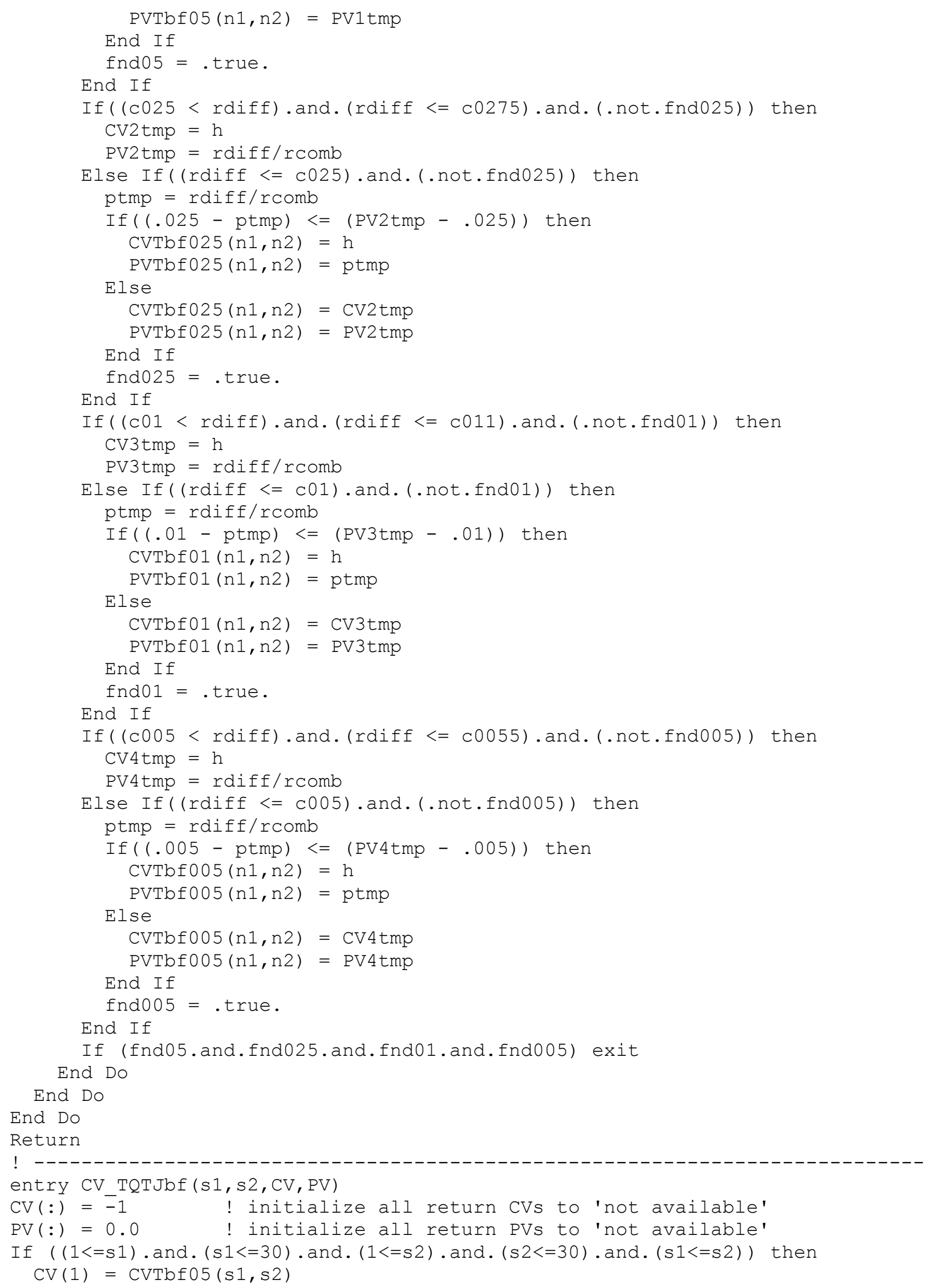




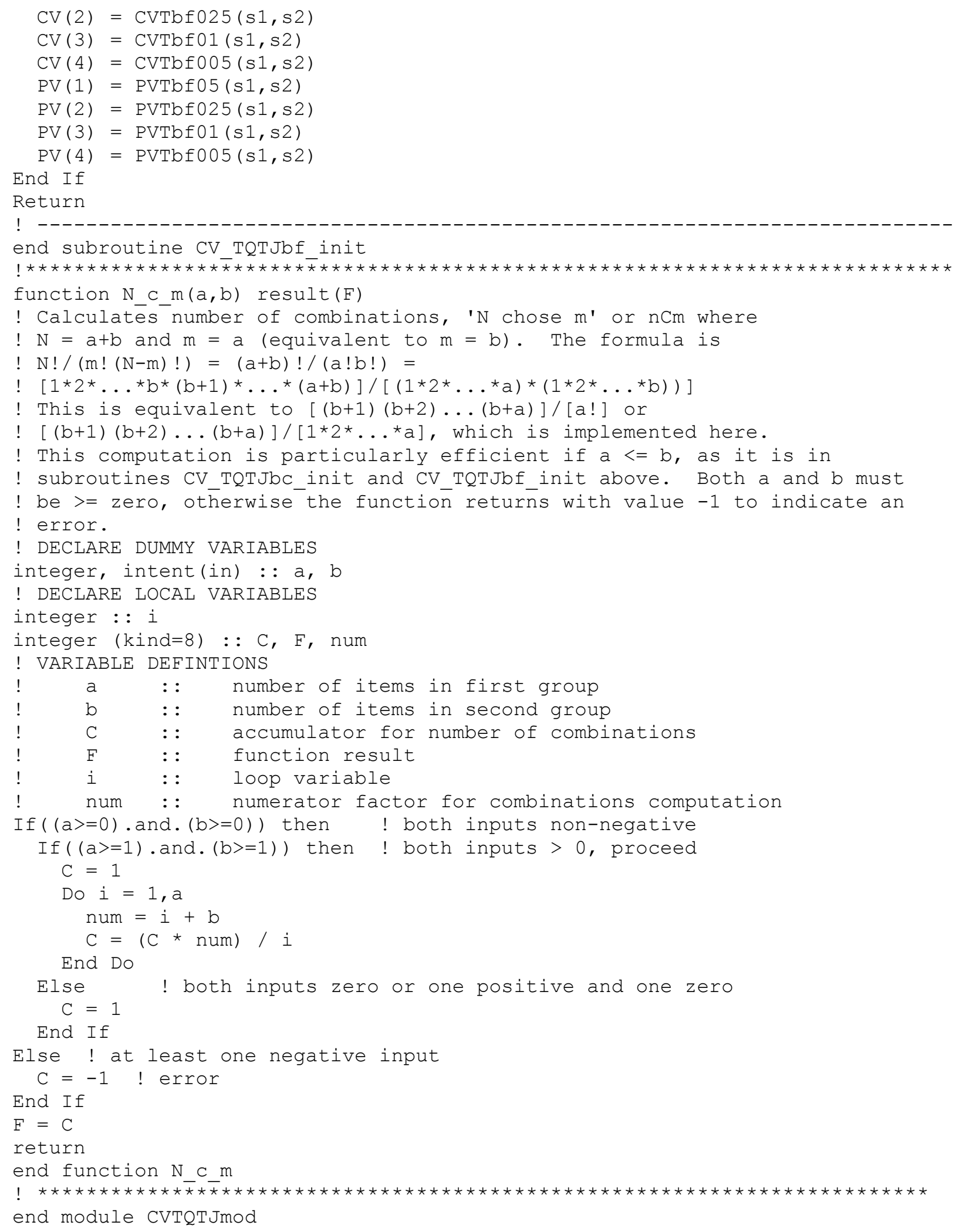




\section{Rosenbaum's Test of Location}

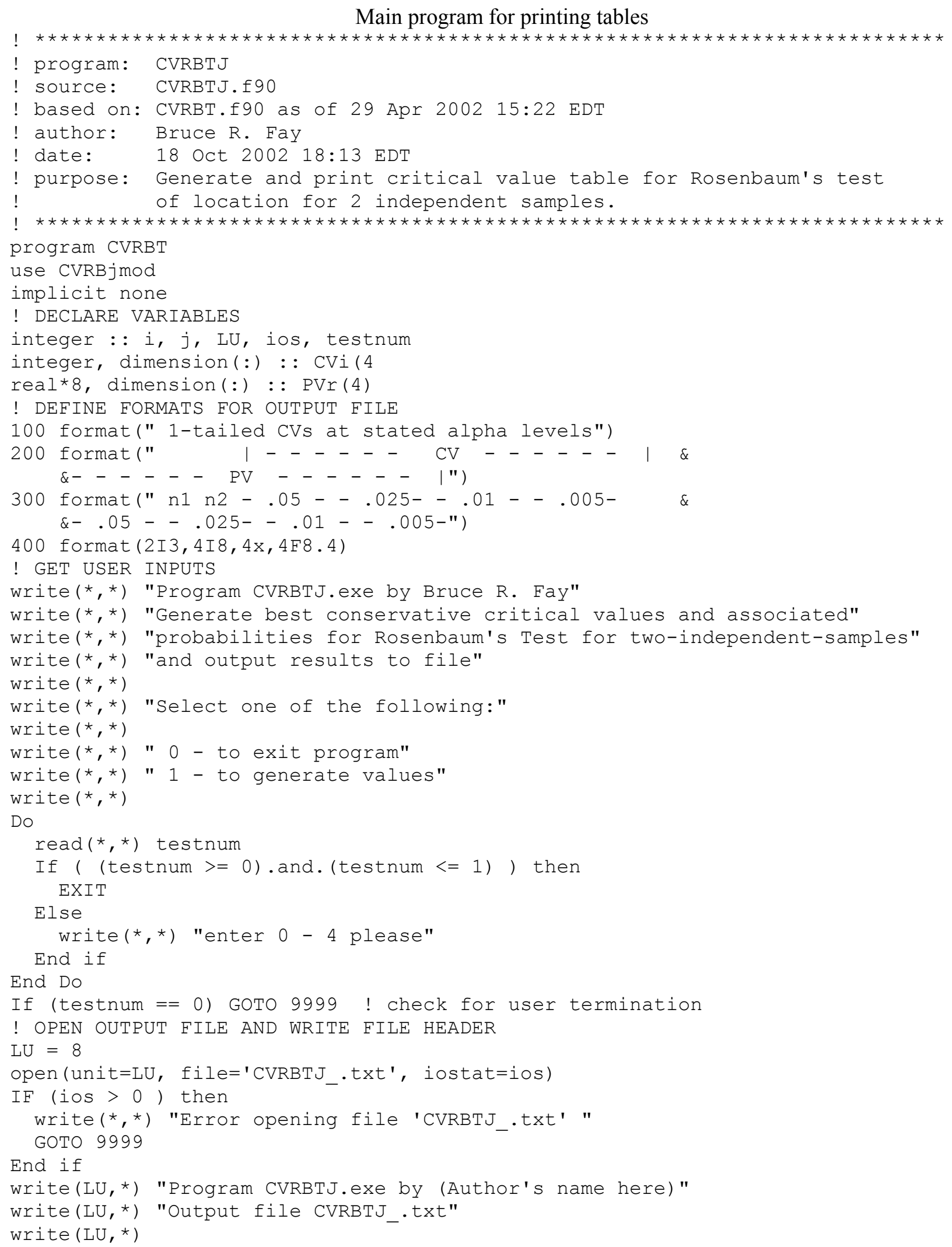




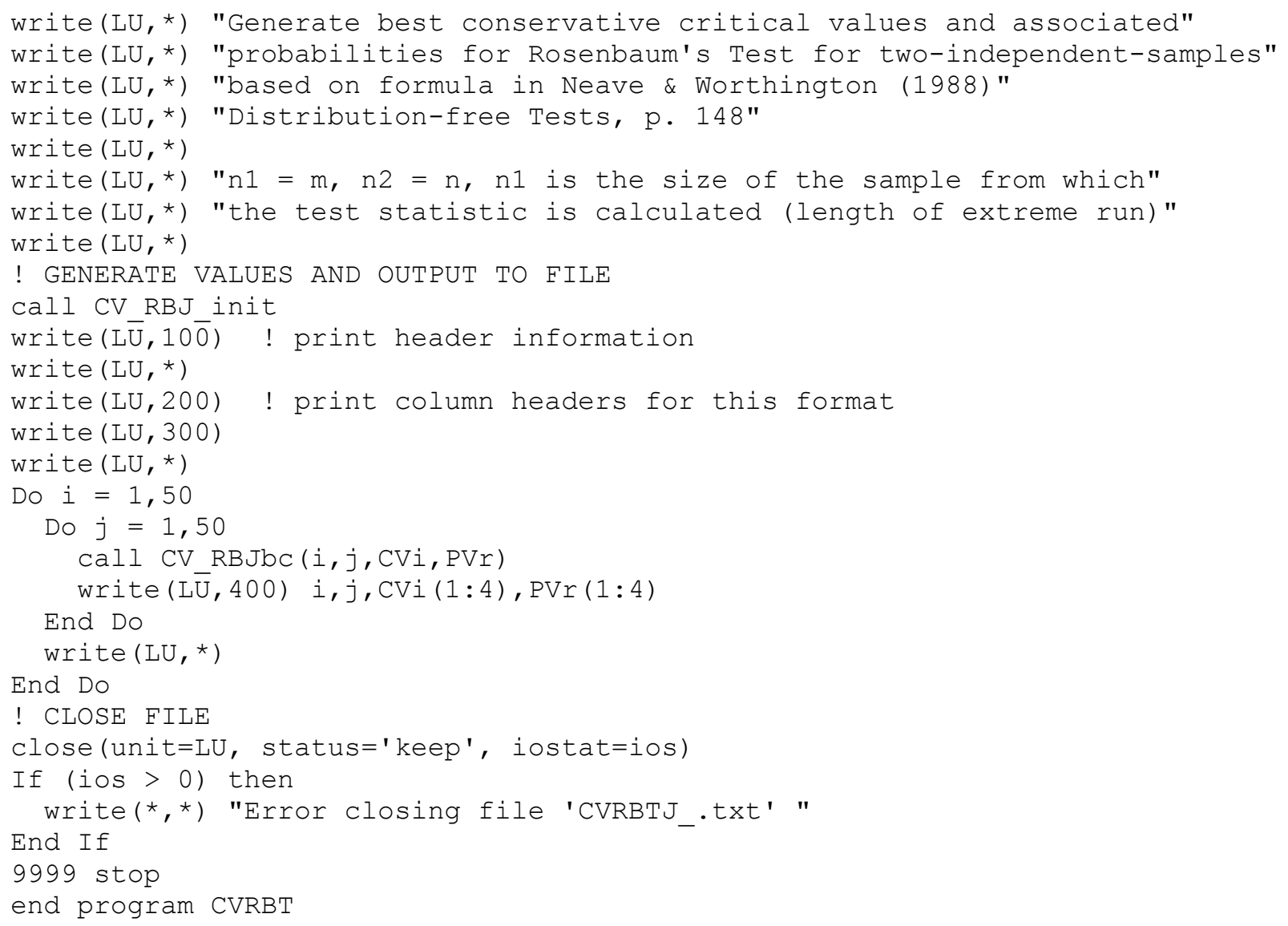

Module for generating critical values and probabilities

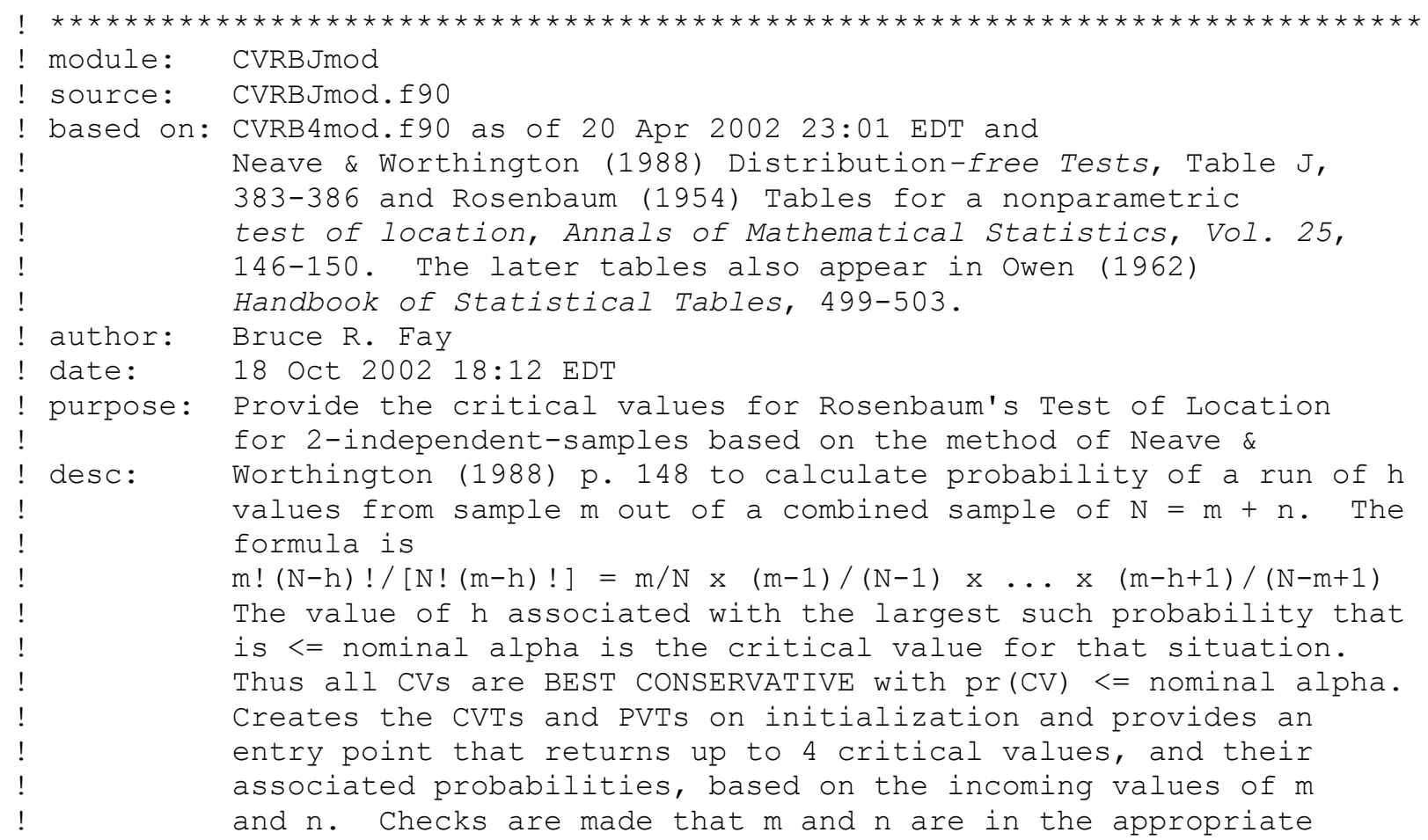




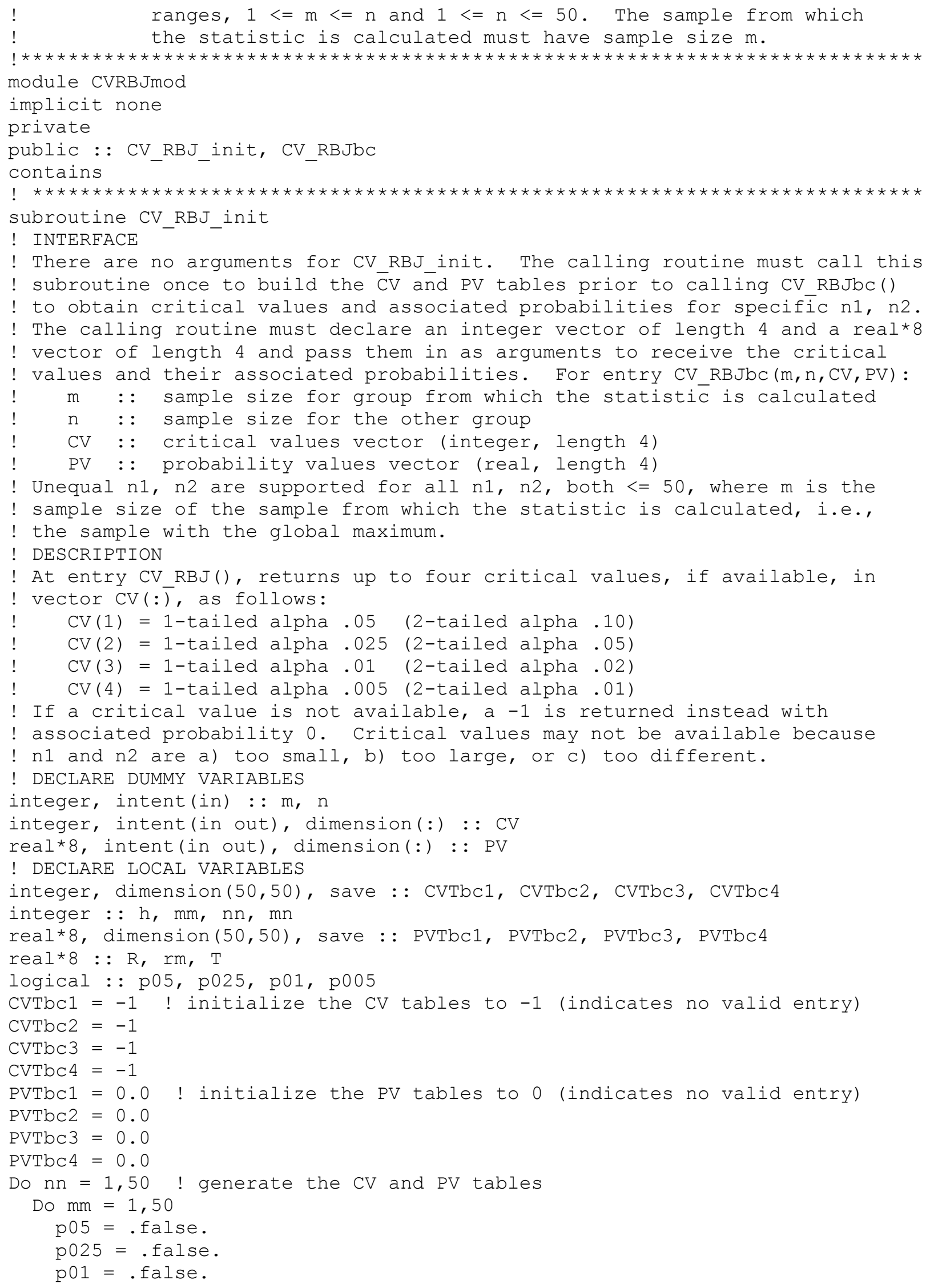




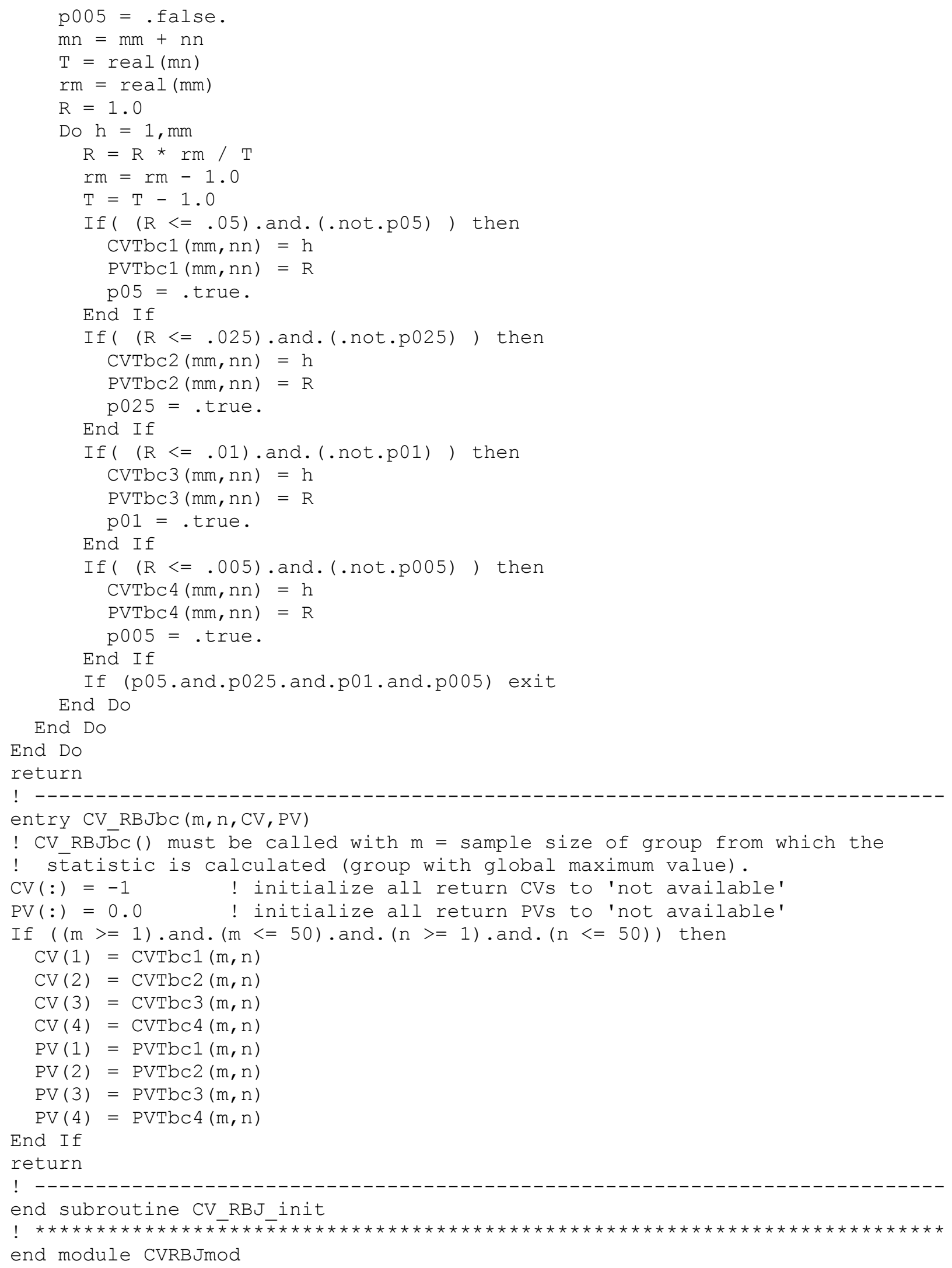




\section{Kolmogorov-Smirnov Test of General Differences}

\section{Main program for printing tables}

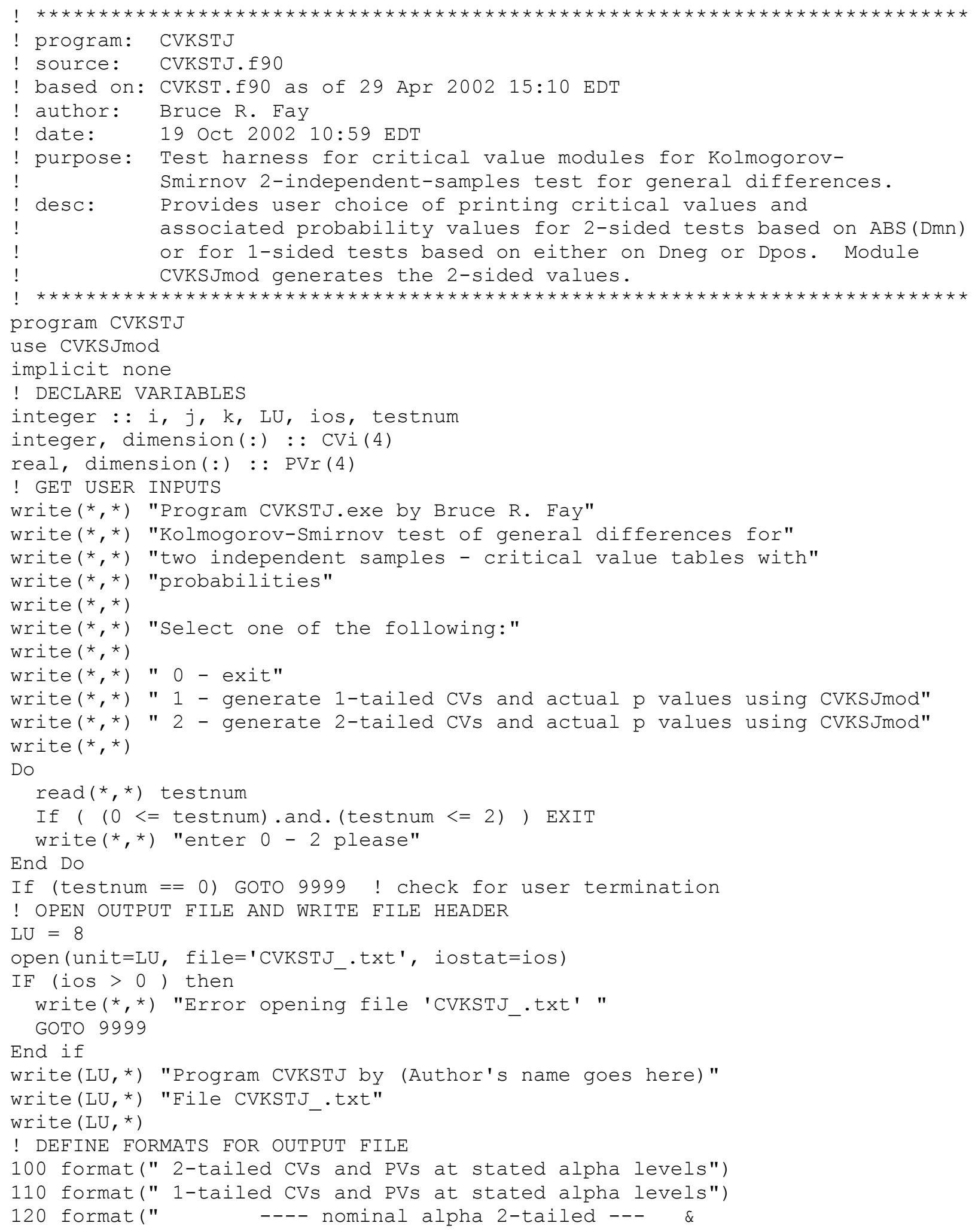




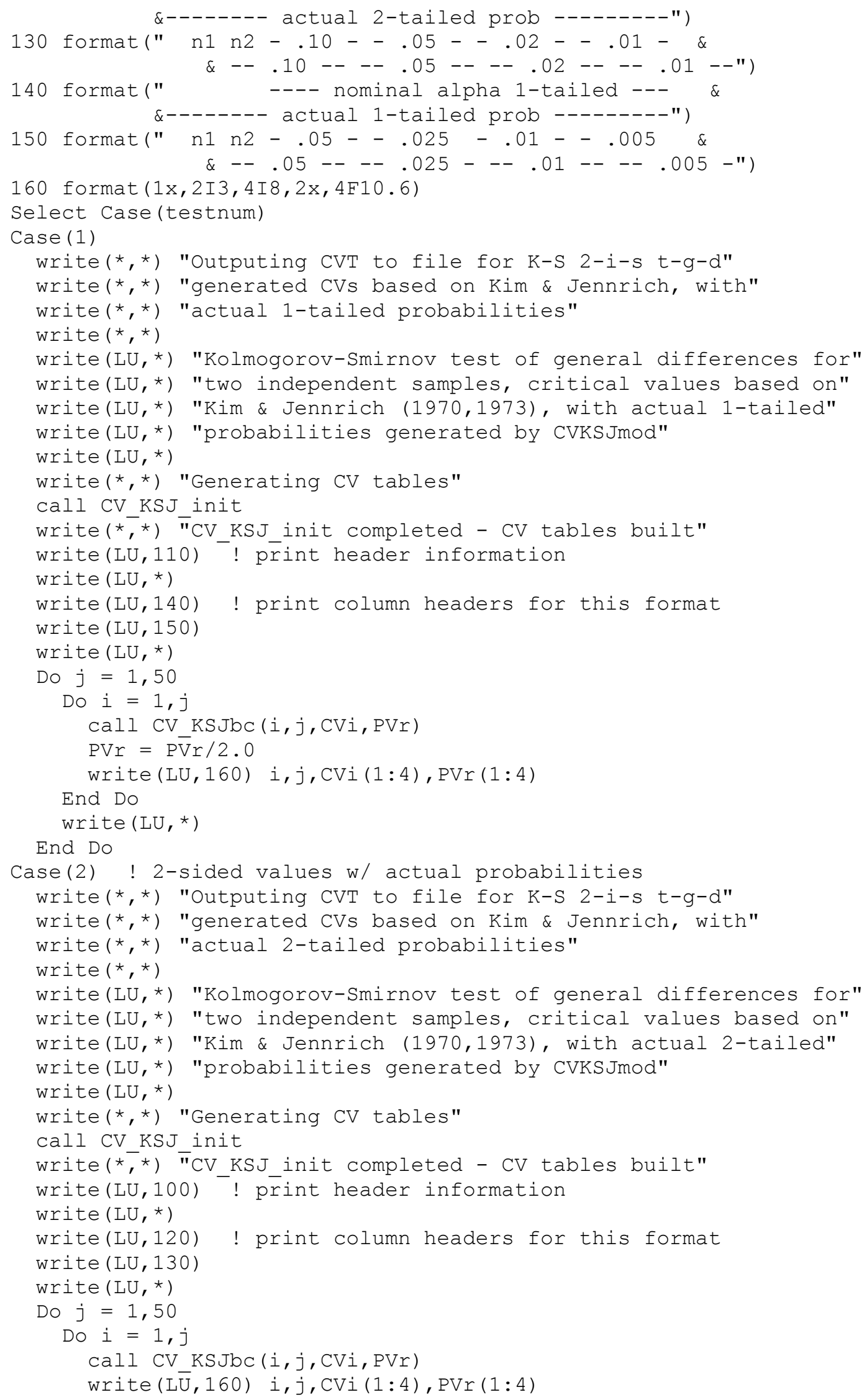




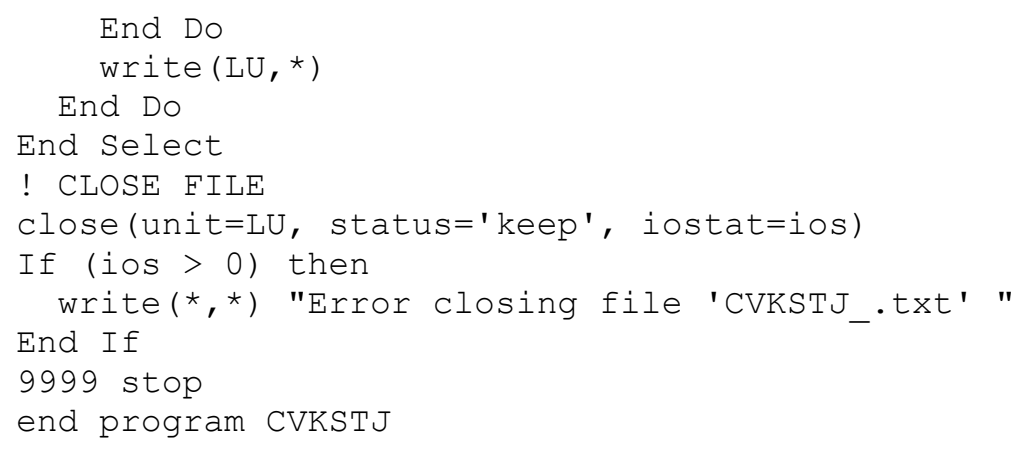

Module for generating critical values and probabilities

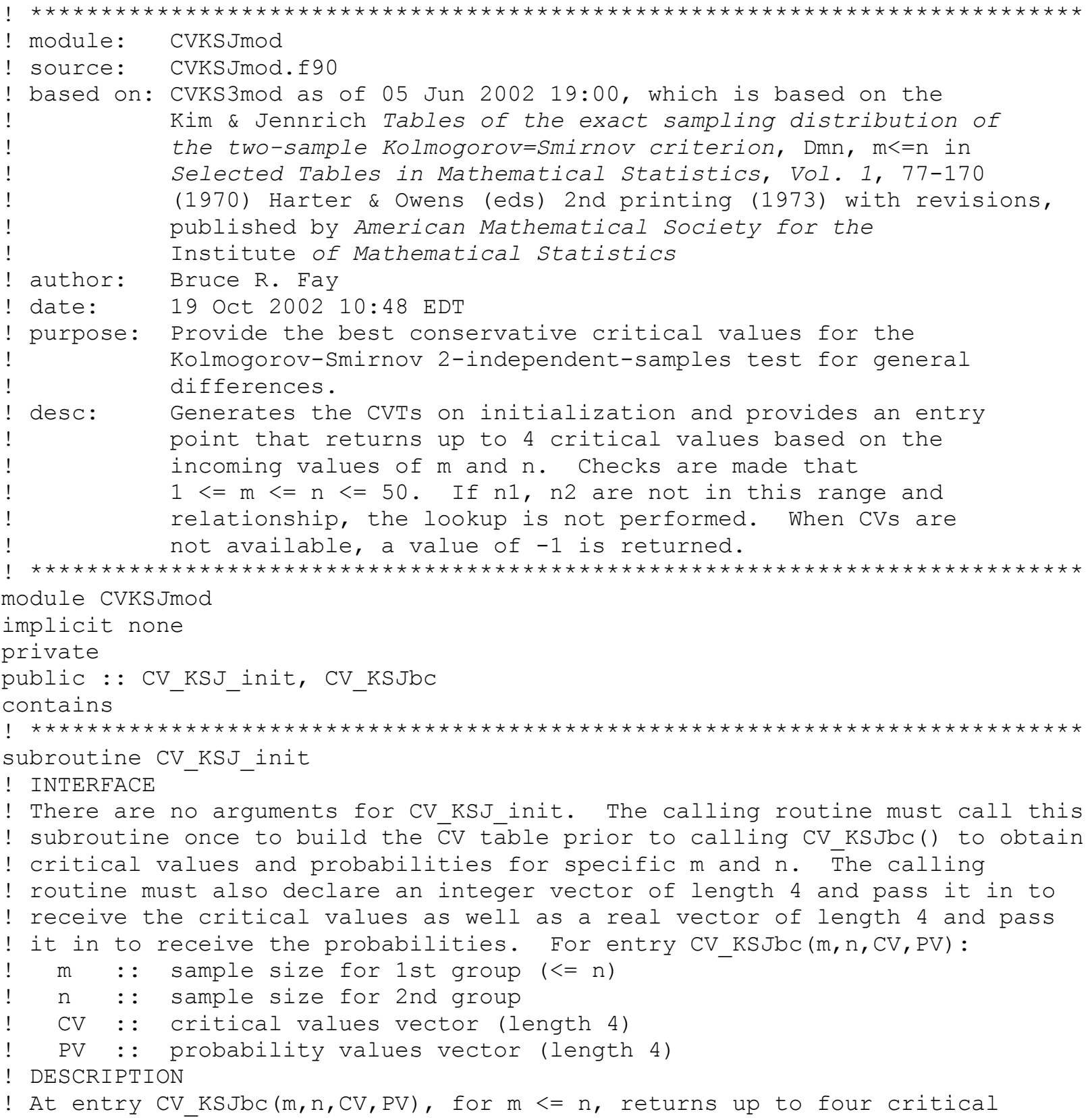




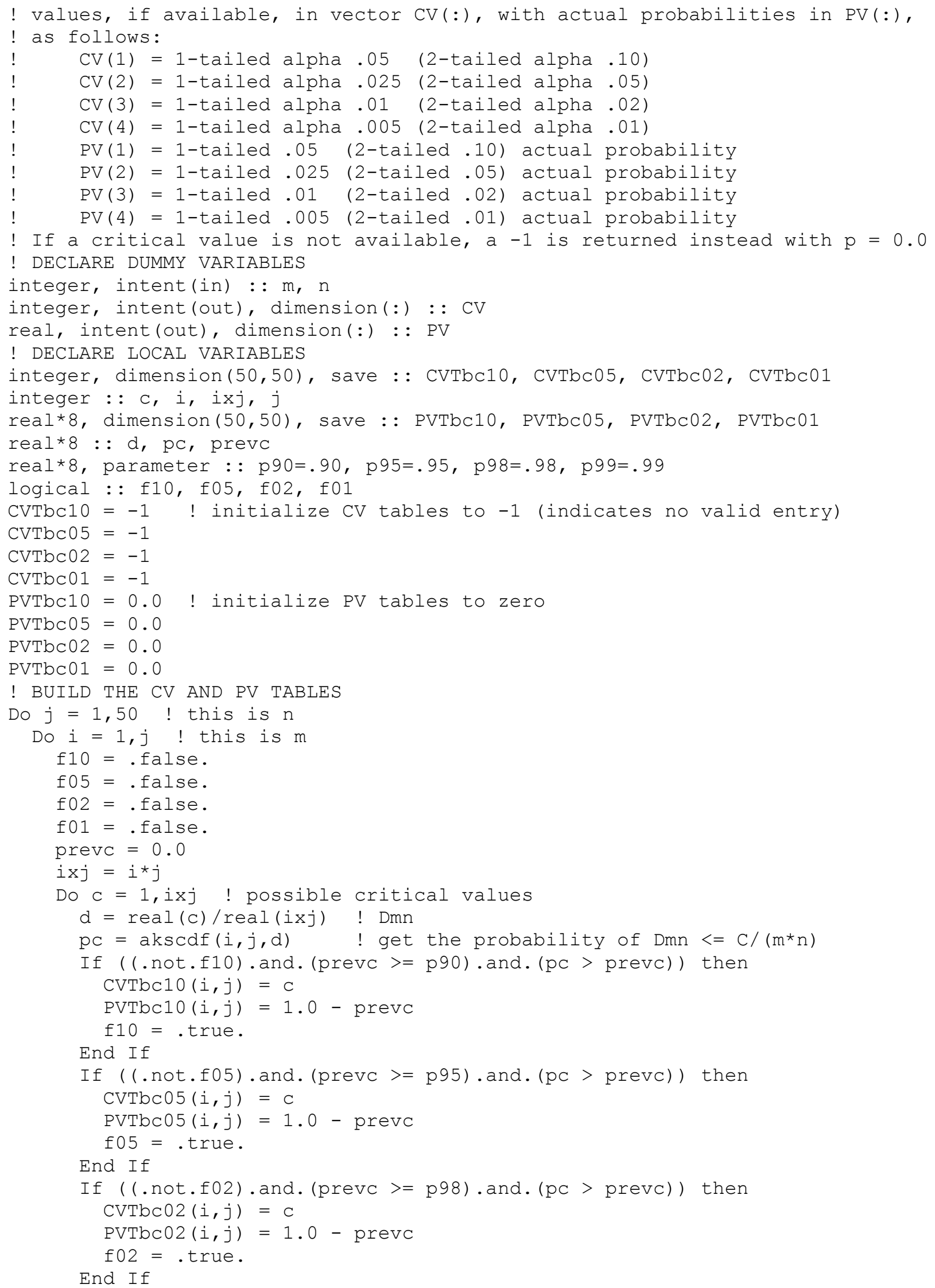




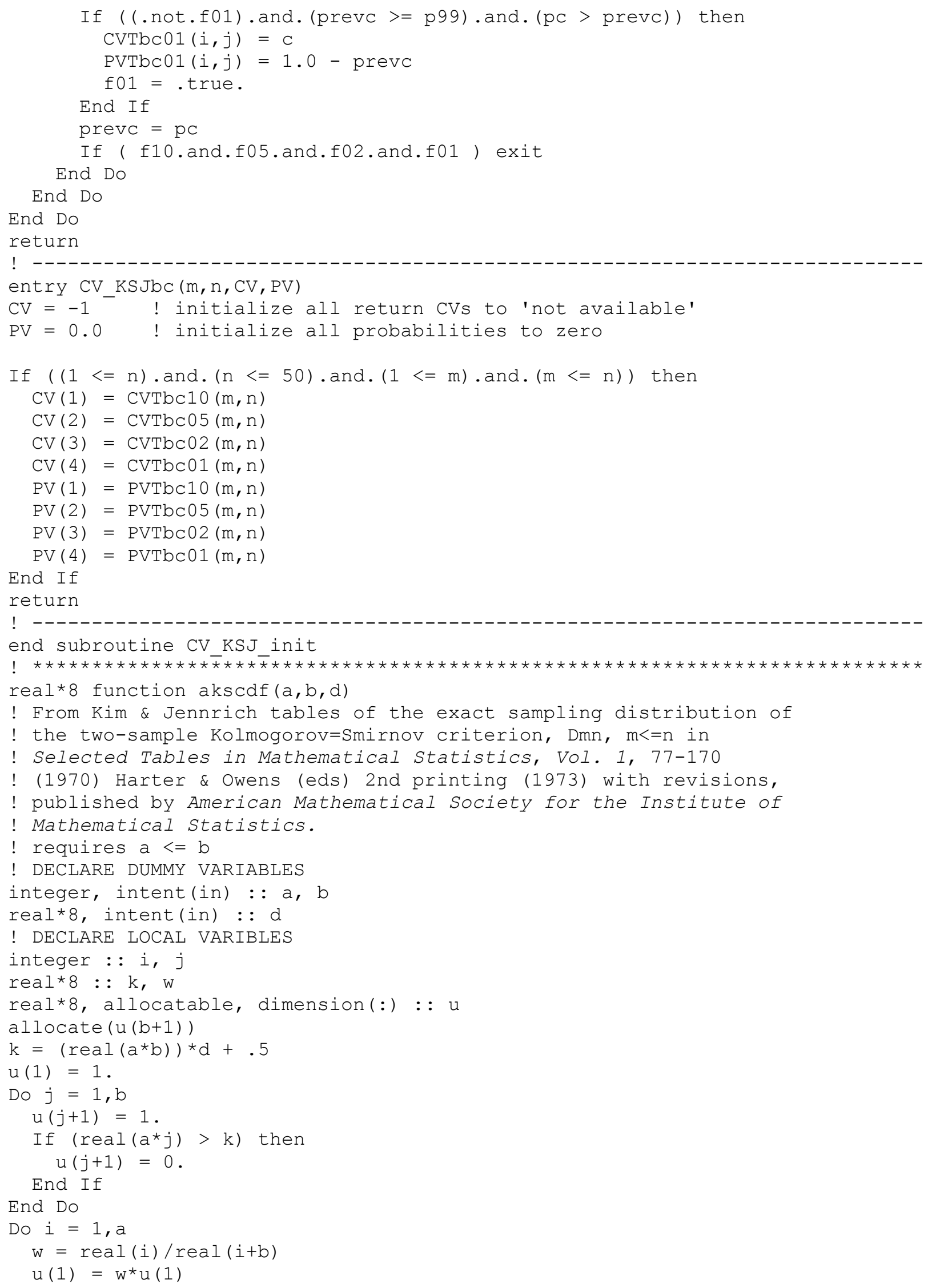




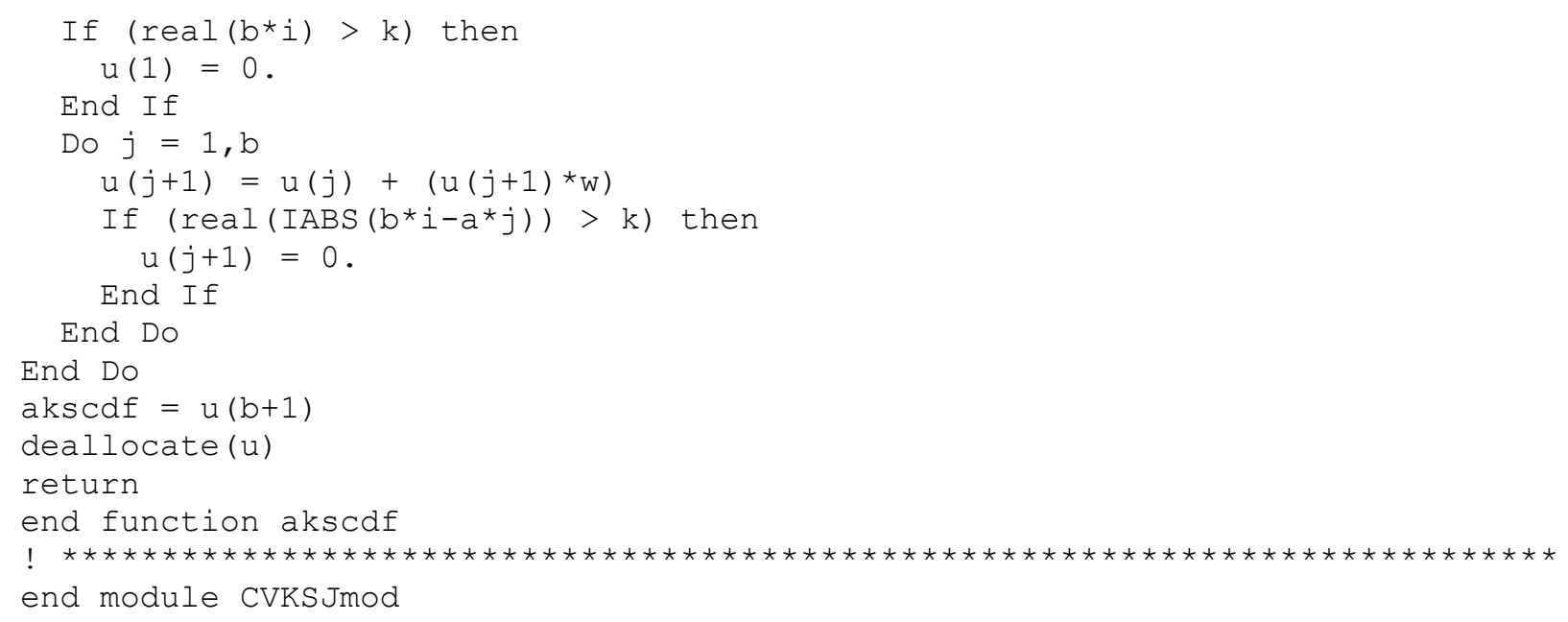

\section{Wilcoxon Rank-sum Test}

\section{Main program for printing tables}

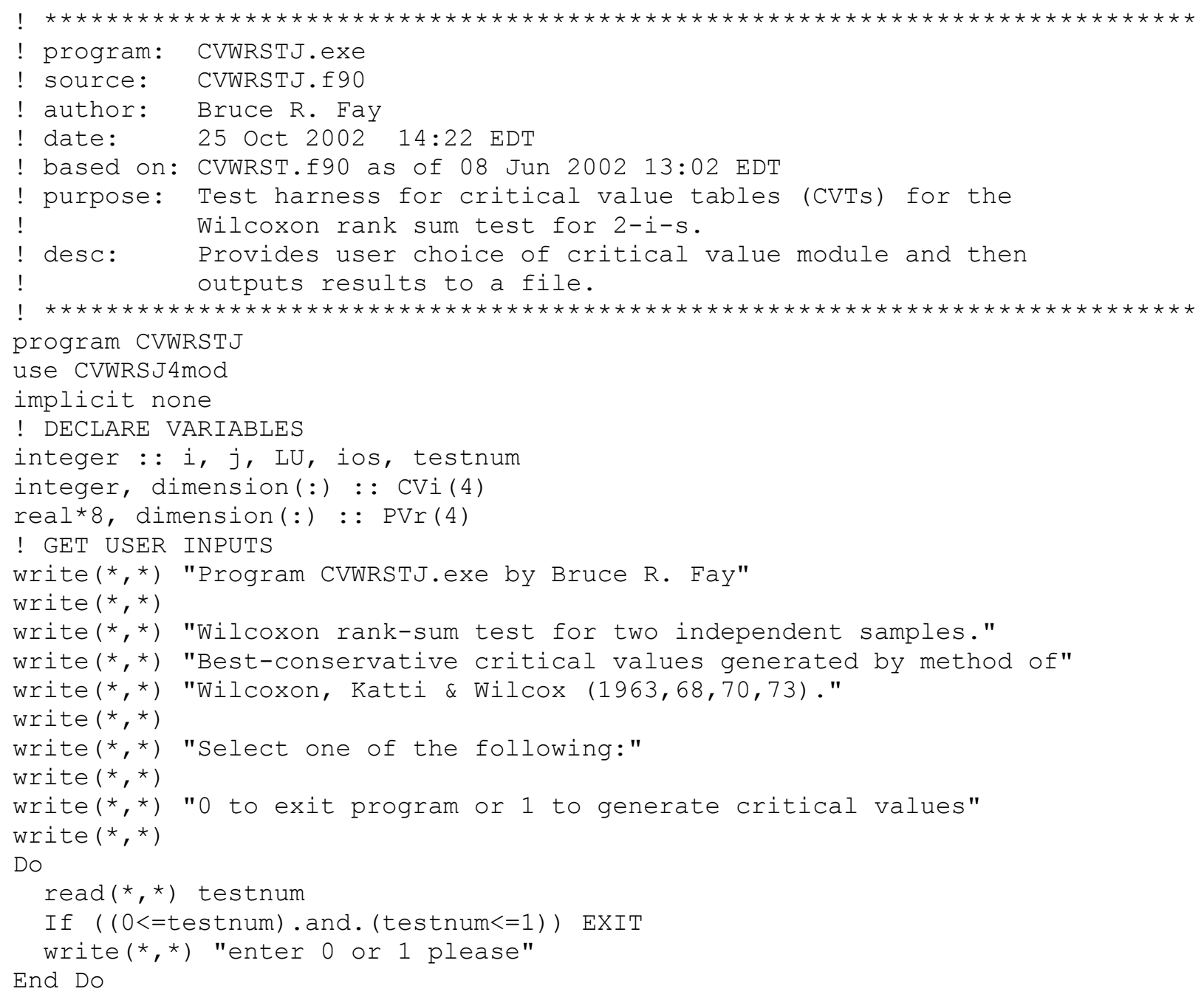




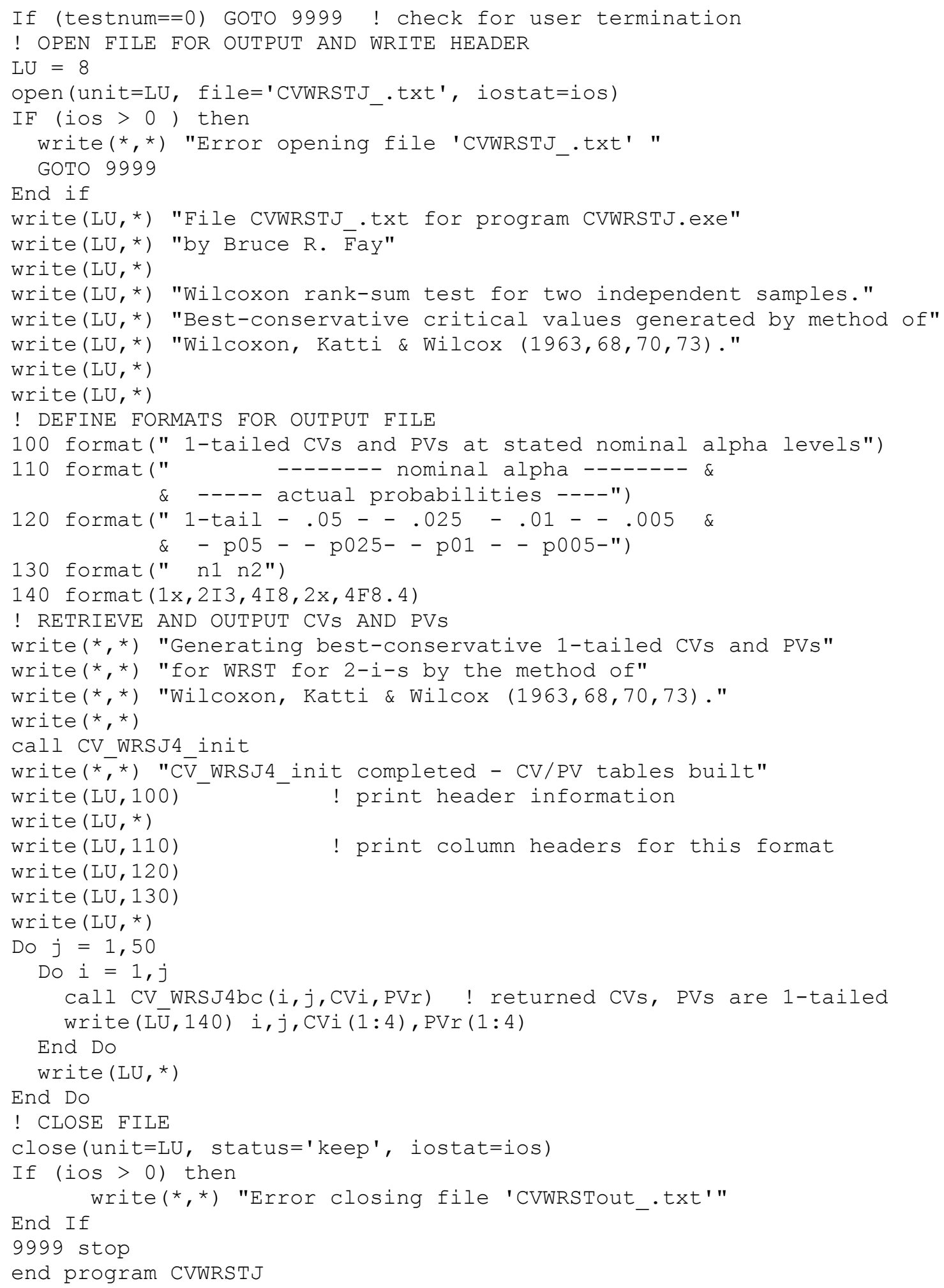




\section{Module for generating critical values and probabilities}

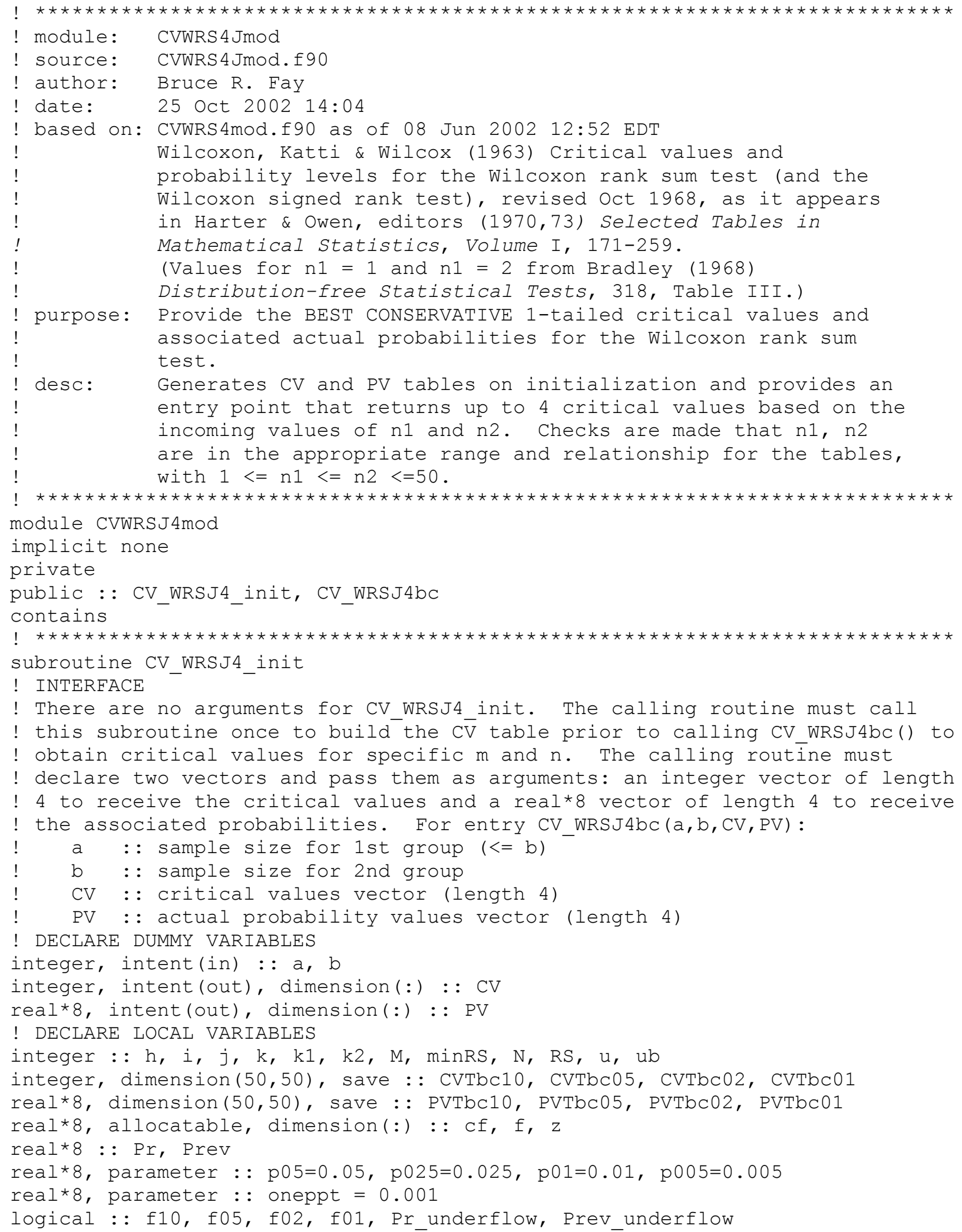




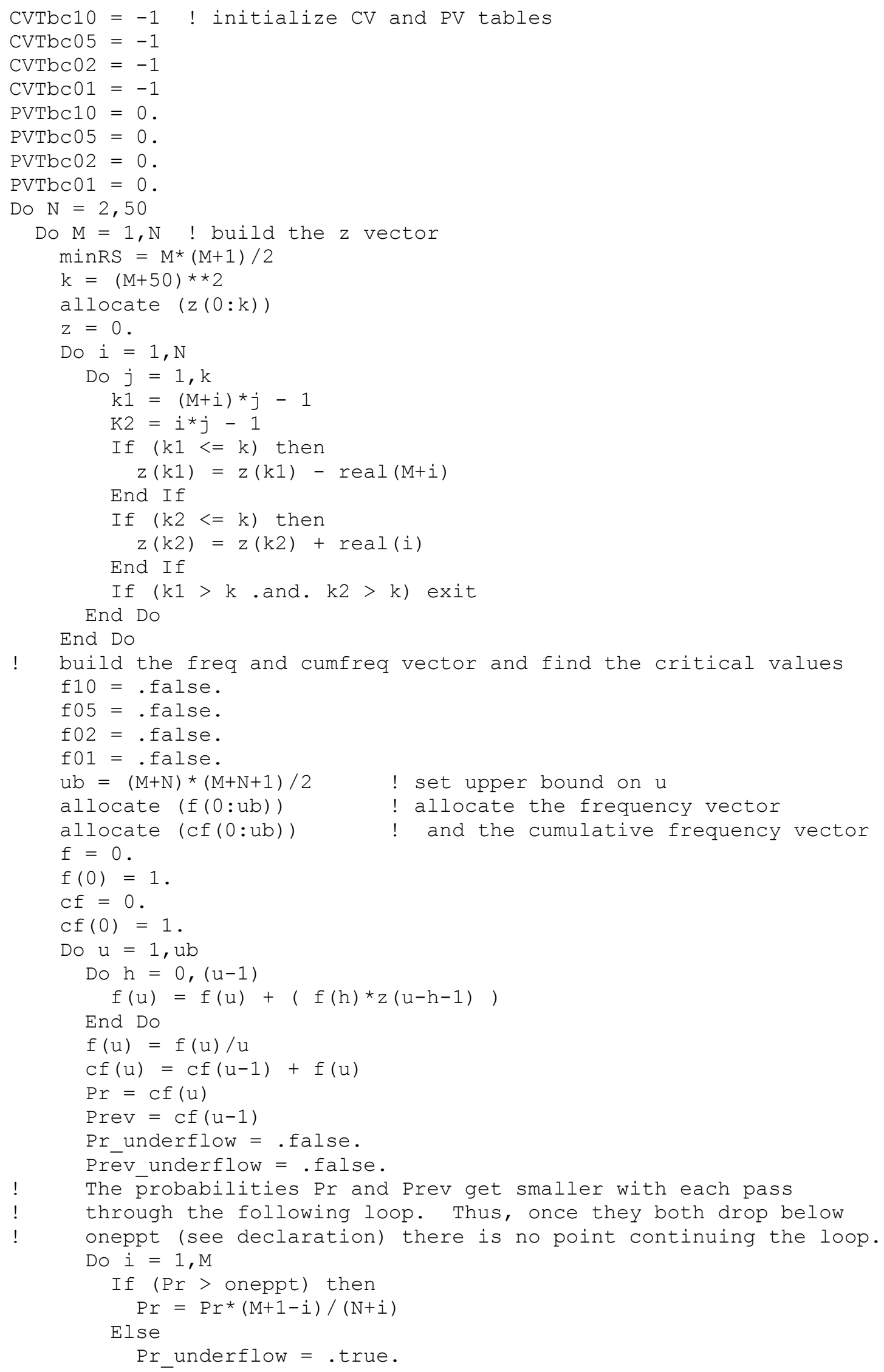




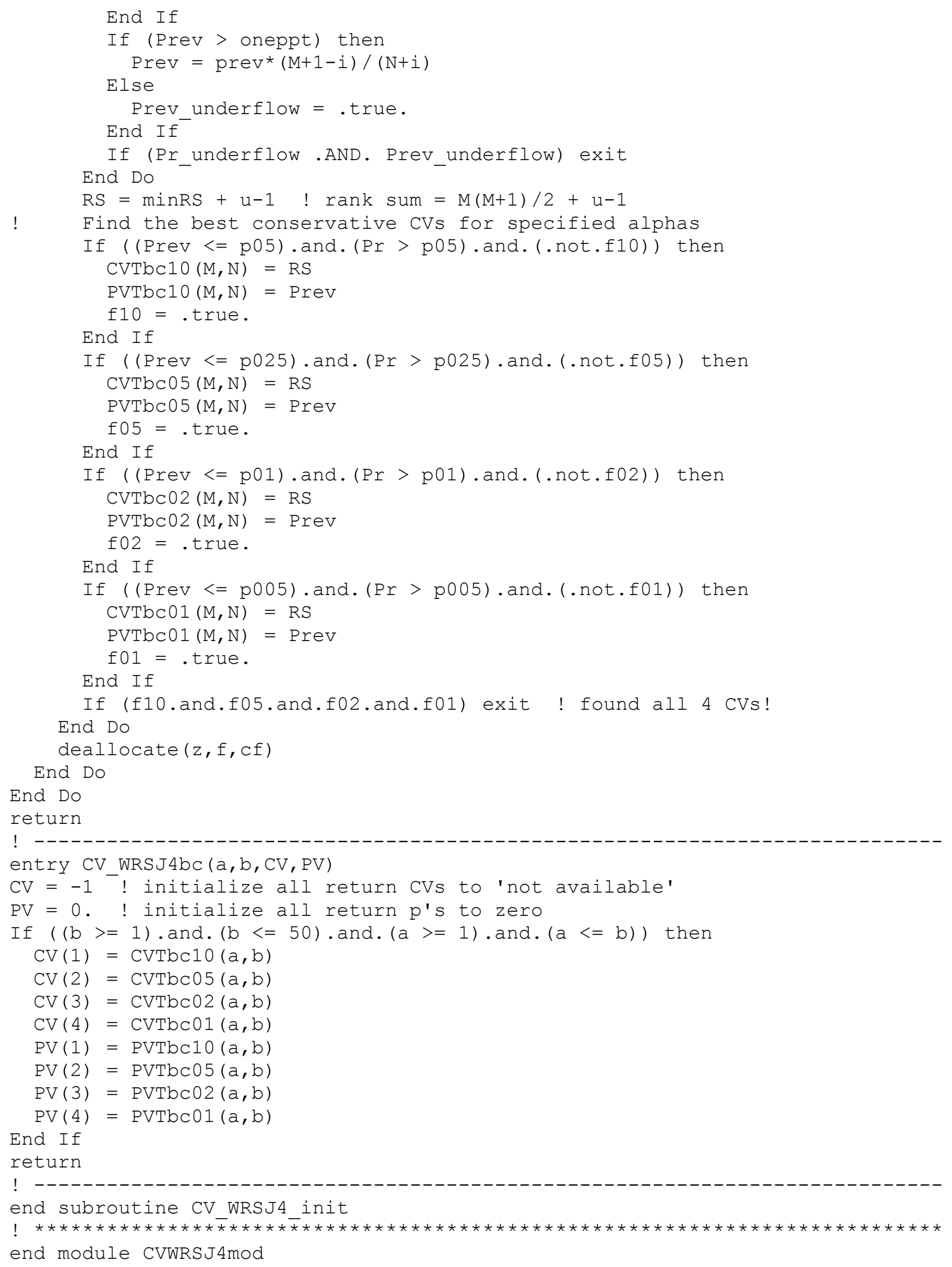

